\title{
Assimilation of All-Sky Infrared Radiances from Himawari-8 and Impacts of Moisture and Hydrometer Initialization on Convection-Permitting Tropical Cyclone Prediction
}

\author{
MASASHI MinAMIDE AND FUQING ZHANG \\ Department of Meteorology and Atmospheric Science and Center for Advanced Data Assimilation and \\ Predictability Techniques, The Pennsylvania State University, University Park, Pennsylvania
}

(Manuscript received 5 December 2017, in final form 20 June 2018)

\begin{abstract}
This study explores the impacts of assimilating all-sky infrared satellite radiances from Himawari-8, a newgeneration geostationary satellite that shares similar remote sensing technology with the U.S. geostationary satellite GOES-16, for convection-permitting initialization and prediction of tropical cyclones with an ensemble Kalman filter (EnKF). This case studies the rapid intensification stages of Supertyphoon Soudelor (2015), one of the most intense tropical cyclones ever observed by Himawari-8. It is found that hourly cycling assimilation of the infrared radiance improves not only the estimate of the initial intensity, but also the spatial distribution of essential convective activity associated with the incipient tropical cyclone vortex. Deterministic convection-permitting forecasts initialized from the EnKF analyses are capable of simulating the early development of Soudelor, which demonstrates encouraging prospects for future improvement in tropical cyclone prediction through assimilating all-sky radiances from geostationary satellites such as Himawari-8 and GOES-16. A series of forecast sensitivity experiments are designed to systematically explore the impacts of moisture updates in the data assimilation cycles on the development and prediction of Soudelor. It is found that the assimilation of the brightness temperatures contributes not only to better constraining moist convection within the inner-core region, but also to developing a more resilient initial vortex, both of which are necessary to properly capture the rapid intensification process of tropical cyclones.
\end{abstract}

\section{Introduction}

Despite decades of effort, accurate prediction of tropical cyclone (TC) rapid intensification (RI) remains challenging. The intensification of TCs has limited predictability since it involves a complex interplay among multiscale dynamics, including but not limited to environmental flow and $\mathrm{TC}$ vortex interaction, air-sea exchange, and mesoscale and microscale convective, microphysical, and radiative processes. Zhang and Sippel (2009) found that multiscale interactions between the chaotic nature of moist convection and vortex flow intrinsically limits the predictability of TCs. Small initial condition errors in the moisture field, together with the influence of environmental conditions such as sea surface temperature and vertical wind shear, induce large variability in the distribution of convection and thus large intrinsic uncertainty in forecasts of TC intensification (Tao and Zhang 2014; Zhang and Tao 2013). As is

Corresponding author: Professor Fuqing Zhang, fzhang@psu.edu also demonstrated by Rappin et al. (2013), for skillful intensification forecasts of TCs, accurate representation of initial atmospheric conditions on a variety of scales with data assimilation is essential. Emanuel and Zhang (2017) found that initialization of tropospheric moisture plays a significant role in the evolution of forecasted TC intensity that adds to the uncertainties from the impact of initial vortex intensity and varying environmental conditions (Emanuel and Zhang 2016).

Recently, assimilation of observational data on the convective scales that are essential to the TC life cycles has been explored. The assimilation of Doppler radar observations with an ensemble Kalman filter using a convection-permitting numerical weather prediction model has demonstrated significant improvement in TC intensity and precipitation forecasts (Zhang et al. 2009, 2011; Aberson et al. 2015; Zhang and Weng 2015). Flight-level Doppler radar and dropsonde observations from airborne surveillance have also been shown to improve forecasts (Wu et al. 2012; Weng and Zhang 2016), but those observations are temporally and spatially 
limited. Because observations over tropical oceans are mainly satellite-based, the utilization of satellite radiance data has great potential in further improving TC forecasts. Given the need and importance of high spatiotemporalresolution observations, the new-generation geostationary satellites - the Advanced Baseline Imager (ABI) on board the Geostationary Operational Environmental Satellite 16 (GOES-16) in 2016 (hereafter GOES-16 ABI), and the Advanced Himawari Imager on the Himawari-8 satellites in 2014 (hereafter Himawari-8 AHI) -were launched. Both new-generation geostationary satellites contain 10 infrared channels with $2 \mathrm{~km} \times 2 \mathrm{~km}$ spatial resolution and produce new images every $10-15 \mathrm{~min}$ utes in their routine surveillance mode, which can be further refined to as high as every minute for targeted areas and targeted storms. The lack of in situ observations enhances the importance of remote sensing data in the northwest Pacific region (Wu et al. 2005), where Himawari-8 AHI mainly monitors.

Many studies have shown positive impacts in assimilating clear-sky brightness temperatures for numerical weather prediction (Wang et al. 2015; Zou et al. 2013; Zou et al. 2015). However, the use of all-sky satellite data has been grossly limited, partly because of the strong nonlinearity of cloud-affected radiances to dynamic and thermodynamic atmospheric profiles. Since the inner core regions of tropical cyclones are typically covered by clouds, existing studies on assimilating satellite radiances for TCs have been mostly focused on the clear-sky observations away from TC cores (e.g., Wang et al. 2015; Zou et al. 2013; Zou et al. 2015). Removal of cloud-affected radiances misses potentially beneficial information from the cloud-covered TC inner-core region. Given the need for cloudy-radiance assimilation, several investigators have already conducted studies to estimate the impact of assimilating all-sky infrared brightness temperatures on numerical weather predictions. Vukicevic et al. (2004) showed that assimilation of radiances from the $10.7-\mu \mathrm{m}$ channel on GOES-9 can be used to eliminate spurious model clouds. Vukicevic and Sengupta (2006) also showed that multichannel radiance assimilation generally leads to better forecasts. Jones et al. $(2013,2014)$ and Otkin $(2010,2012)$ conducted observing system simulation experiments (OSSEs) with synthetic all-sky GOES-16 ABI infrared radiances to estimate their potential impact on the prediction of severe weather events. These cloudy radiances provide information on the vertical structure of the moisture field and cloud concentrations (Jones et al. 2014). Zhang et al. (2016, hereafter ZMC16) demonstrated improved TC inner-core initialization following the assimilation of all-sky infrared satellite radiances through a series of convection-permitting OSSEs and GOES-13 real-data assimilation experiments.

In this study, we investigate the rapid-intensification stages of Typhoon Soudelor (2015). Soudelor was the thirteenth named typhoon in northwestern Pacific Ocean in 2015. A few weeks after Himawari-8 started operational monitoring (7 July 2015), Soudelor developed from persistent deep convection over a consolidating low-level circulation center located approximately $400 \mathrm{~km}$ north of Kwajalein Atoll in the Marshall Islands. The Joint Typhoon Warning Center (JTWC) issued a TC formation alert on the tropical depression system on 30 July 2015. Propagating westward, Soudelor reached its maximum intensity of approximately $907 \mathrm{hPa}$ around 4 August, according to the JTWC best track estimate, and was the strongest typhoon in northwestern Pacific Ocean in 2015. Soudelor later caused tremendous damage as it made landfall first on Taiwan and then southern China. The track and intensity of Soudelor from genesis to landfall in Taiwan are depicted in Fig. 1. Soudelor's entire life cycle was located within the coverage of Himawari-8 and observed by full-disk scan with a 10-min temporal frequency.

This study is a follow-up of ZMC16's investigations with observing system simulation experiments that have shown the capability of capturing detailed asymmetric structure of TCs, such as primary rainbands, an eye, and even individual convective clouds, through all-sky infrared radiance assimilation. The purpose of this study is to assess how the improved representations of those structures contribute to the prediction of TCs. This article is organized as follows. Model and experimental designs are described in section 2 . The performance of assimilating observed brightness temperatures by Himawari-8 is given in section 3 . The sensitivity experiments to highlight the impacts of moisture and vortex initializations are discussed in section 4. Concluding remarks are provided in section 5.

\section{Methodology and experimental design}

\section{a. Forecast and radiative transfer models: WRF and CRTM}

This study uses the Advanced Research version of the Weather Research and Forecasting (WRF-ARW) Model, version 3.6.1. WRF-ARW is a fully compressible, nonhydrostatic mesoscale model (Skamarock et al. 2008). Our model configuration uses three two-way nested domains with horizontal grid spacing of 27,9 , and $3 \mathrm{~km}$, which contain $378 \times 243,297 \times 297$, and $297 \times 297$ grid points, respectively. A moving nest is used for the finer two domains (D2 and D3) to follow the center of the TC vortex (Fig. 1). All domains use 61 vertical levels with the model 


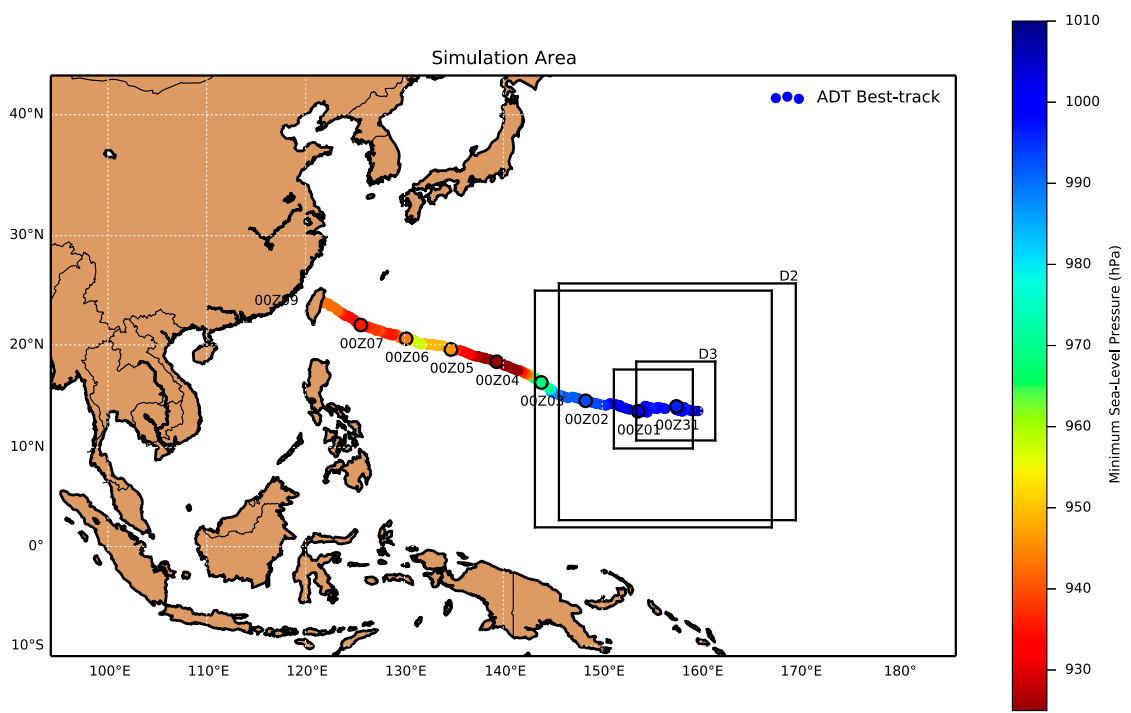

FIG. 1. Simulation area of three nested domains and the track and intensity of Supertyphoon Soudelor (2015) from ADT best track data (colored dots). The positions of moving nested inner domains (D2 and D3) at the initial (1200 UTC 31 Jul 2015) and last (1800 UTC 31 Jul 2015) assimilation cycles are depicted.

top set at $10 \mathrm{hPa}$ in a stretched vertical grid. The WRF single-moment 6-class mixed-phase microphysics scheme (WSM6) (Hong and Lim 2006), the Yonsei University planetary boundary scheme (Hong et al. 2006), and the Rapid Radiative Transfer Model (RRTM) longwave and shortwave radiation schemes (Iacono et al. 2008) are applied for all domains. The Tiedtke (1989) cumulus parameterization scheme was only applied to the coarsest $(27 \mathrm{~km})$ domain.

We use the Community Radiative Transfer Model (CRTM: Han et al. 2006, 2007; Weng 2007) as the forward model to convert between the model and observation space. CRTM is a rapid forward radiative transfer model developed by Joint Center for Satellite Data Assimilation (JCSDA). The simulated brightness temperatures are computed with the successive order of interaction (SOI) forward solver (Heidinger et al. 2006) using the OPTRAN code from CRTM.

\section{b. Data assimilation system: WRF-EnKF}

As in ZMC16, we use the ensemble Kalman filter (EnKF) data assimilation system (Zhang et al. 2009, 2011; Weng and Zhang 2012, 2016; ZMC16) developed at The Pennsylvania State University (PSU), which is built around WRF-ARW and CRTM. This CRTM-integrated data assimilation framework enables us to directly assimilate all-sky brightness temperatures (BTs) with high temporal and spatial resolution into the PSU WRF-EnKF analysis and forecast system. The covariance relaxation method of Zhang et al. (2004) with coefficient $=0.75$ is applied. For simplicity and for preparation of more efficient implementation in future real-time applications, we assimilate only one of three water vapor channels of Himawari-8 AHI (channel 8: wavelength is $6.19 \mu \mathrm{m}$ ) every $1 \mathrm{~h}$ (which is slightly different from the OSSEs in ZMC16 that used all three water vapor channels assimilated every $10 \mathrm{~min}$ ). Channel 8 is sensitive to upper-level moisture in the clear-sky regions and to the cloud tops in the cloudy regions. Because the three water vapor channels are strongly correlated (with correlation coefficient $>0.9$, particularly in the cloudy regions that covers most of D3), assimilation of the other two water vapor channels is expected to produce similar results. Observed BTs are first interpolated to fit the forecast model grid and assimilated assuming a minimum observation error of $3 \mathrm{~K}$. To alleviate large representativeness errors when assimilating all-sky BTs-especially relevant to cloudaffected BTs-we employ the adaptive observation error inflation (AOEI) method used in ZMC16 and Minamide and Zhang (2017). By using AOEI, we adaptively estimate the observation error variance $\sigma_{o, \mathrm{AOEI}}^{2}=\max \left\{\sigma_{o t}^{2},\left[y_{o}-H\left(x_{b}\right)\right]^{2}-\sigma_{b}^{2}\right\}$, where $y_{o}$ represents the observations, $H\left(x_{b}\right)$ the simulated observations, $\sigma_{b}^{2}$ the background error variance, and $\sigma_{o}$ the uniform-distributed uncorrelated observation error. As in the OSSEs of Minamide and Zhang (2017), we assume the value of $3 \mathrm{~K}$ as uniform-distributed uncorrelated observation error composed of instrument noise and flow-independent component of representative error, which is consistent with other studies such as Otkin (2012) and Honda et al. (2018). Following the AOEI 
algorithm, each observation is either assigned an error of $3 \mathrm{~K}$ or an adaptively inflated value for the observation error. Similar to Minamide and Zhang (2017), the successive covariance localization (SCL) method proposed by Zhang et al. (2009) is applied in this study. SCL is designed to capture both convective-scale and largescale structures with a combination of small and large covariance localization distance. Also similar to ZMC16 and Minamide and Zhang (2017), we assimilate each single BT observation that is thinned to the center of a $12 \mathrm{~km} \times 12 \mathrm{~km}$ box with a $30-\mathrm{km}$ radius of influence, and each single BT observation that is thinned to the center of a $18 \mathrm{~km} \times 18 \mathrm{~km}$ box with a $200-\mathrm{km}$ radius of influence in the finest domain. The observation density of $12-\mathrm{km}$ spacing is chosen to resolve the minimum model effective horizontal scale $(4 \mathrm{~km} \times 3 \mathrm{~km}$ grid spacing model domain) to capture the convective-scale structures. The other observation density of every $18 \mathrm{~km}$ in spacing is chosen to balance the total number of the observations for large-scale structures to be about the half of observations for updating convective-scale structures, which is the ratio originally and empirically used in Zhang et al. (2009). For the covariance localization radius, we used $30 \mathrm{~km}$ for smaller scale because it minimized the rootmean-square error (RMSE) of hydrometeor fields that are more directly related to the convective activity (not shown; the OSSE experimental design can be found in ZMC16). We did not see a clear dependence of RMSE on the localization radius for other variables. Thus, here, we use $200 \mathrm{~km}$ following Otkin (2012), ZMC16, and Honda et al. (2018). We acknowledge that the specification and selection of the observation error and density, as well as the covariance localization, are largely empirical in nature. All of these should be further systematically explored in the future to maximize the benefit of assimilating high spatiotemporal resolution geostationary all-sky satellite radiance for TC analysis and prediction.

\section{c. Experimental design}

The National Centers for Environmental Prediction Global Forecast System Final Analysis (NCEP GFS FNL) is used to generate the initial and boundary conditions. The use of FNL analysis is to focus the study on the initial condition uncertainty in tropical cyclone prediction with a limited-area model. A 60 -member ensemble is initiated at 0000 UTC 31 July by adding perturbations derived from an application of WRF's three-dimensional variational data assimilation (3DVar) using the cv5 flowdependent background error covariance option (Barker et al. 2004) to the 3-day forecast initialized from FNL at 0000 UTC 28 July 2015 . The ensemble is integrated for $12 \mathrm{~h}$ to $1200 \mathrm{UTC} 31 \mathrm{July}$ and used as an initial input for
WRF-EnKF. As in ZMC16, a benchmark WRF-EnKF experiment assimilates minimum sea level pressure at the TC center position [derived from the advanced Dvorak technique (ADT); Olander and Velden 2007] every hour until 1800 UTC 31 July, hereafter referred to as hurricane position and intensity (HPI). ${ }^{1}$ The second experiment assimilates clear-sky BTs in addition to HPI, hereafter referred to as the clrBT+HPI experiment. The third experiment assimilates all-sky BTs (under both clear-sky and cloudy-sky conditions) and HPI, hereafter referred to as BT $+\mathrm{HPI}$ experiment. Every $6 \mathrm{~h}$ during the assimilation cycles, boundary and environmental conditions (i.e., outside of the $600-\mathrm{km}$ circle from TC center) are blended with the perturbed FNL analysis with the transition zone that linearly increase the weight of the FNL analysis from zero at radius $600 \mathrm{~km}$ to 1 at radius $900 \mathrm{~km}$ and beyond. The relaxation to the global analysis is again designed to focus the study on the impacts of all-sky radiance assimilation to the tropical cyclone inner-core region. Deterministic forecasts from the EnKF (ensemble mean) analysis are performed every $3 \mathrm{~h}$ until 0000 UTC 5 August to simulate the development and intensification of Soudelor. The deterministic forecast from 1800 UTC 31 July for the $\mathrm{BT}+\mathrm{HPI}$ assimilation experiment is designated as the control run for the following sensitivity experiments, and hereafter referred to as CNTL.

To differentiate the clear-sky BTs from cloud-affected BTs in the clrBT + HPI experiment, we follow the strategy that defines a threshold for the corresponding BT, denoted as $\mathrm{BT}_{\text {lim }}$, as proposed by Harnisch et al. (2016). The impact of clouds on BT is quantified by calculating the difference between simulated first-guess BT and simulated pseudocloud-free BT model equivalent that turns off the cloud scattering and emission in the radiative transfer. Figure 2 exhibits the average impacts of the presence of clouds as a function of the first-guess BT, computed with the CNTL deterministic forecast. For channel 8 , the average impact roughly increases linearly for first-guess with low BTs, while remaining around zero with high BTs. In this study, we define the $\mathrm{BT}_{\lim }$ for channel 8 as the BT where the linear regression line calculated with BT $<225 \mathrm{~K}$ crosses the $y=0$ line, which is shown as the dotted vertical line in Fig. 2. Although not assimilated, the $\mathrm{BT}_{\lim }$ values for channels 9 and 10 are similarly calculated from their respective linear regression lines for BT $<235$ and $245 \mathrm{~K}$, respectively. Figure 2 shows

\footnotetext{
${ }^{1}$ Note that Soudelor was a tropical cyclone over the western Pacific Ocean, which is classified as a "typhoon," not a "hurricane." Nevertheless, we used "hurricane position and intensity" or "HPI" in this study to keep the consistent terminologies with the previous studies that also assimilated minimum SLP (e.g., Zhang et al. 2016; Minamide and Zhang 2017).
} 


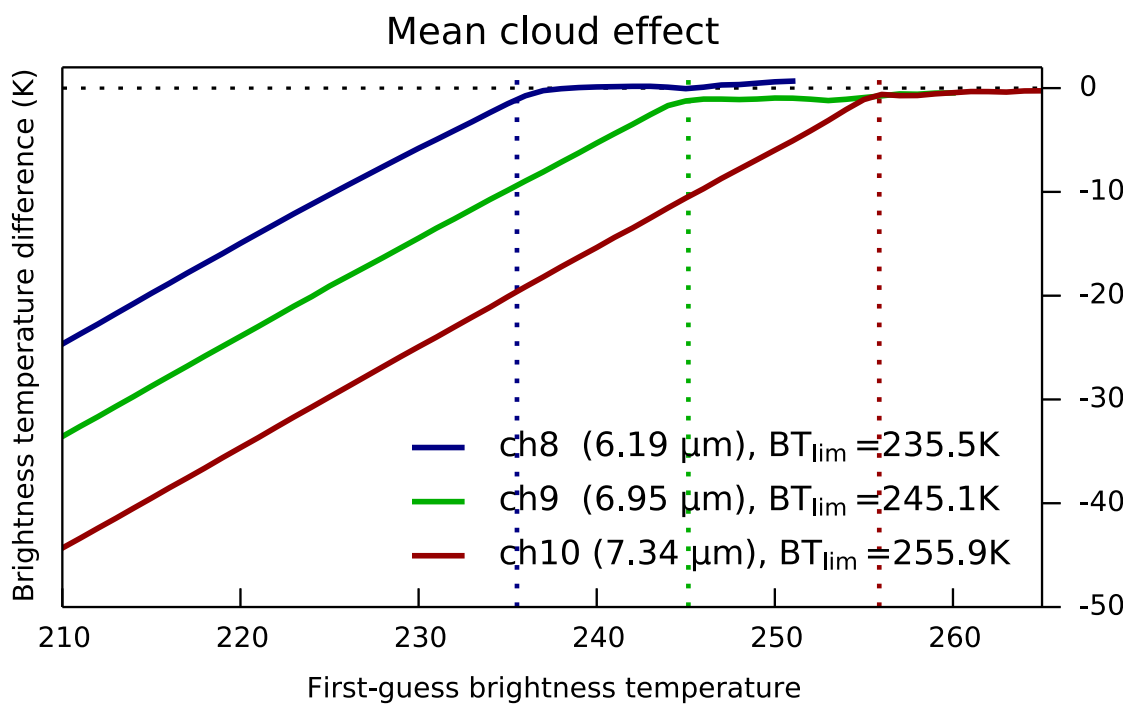

FIG. 2. Average of brightness temperature differences of EnKF mean background equivalent without and with cloud scattering and cloud emission for water vapor channels from Himawari-8 AHI (color coded). The vertical dashed lines show the selected values of $\mathrm{BT}_{\text {lim }}$.

that the $\mathrm{BT}_{\text {lim }}$ well separates the BTs whose value is reduced by the presence of clouds and the BTs without significant impacts of clouds. In clrBT+HPI, we assimilate the BTs only where both the observation and model prior are greater than $\mathrm{BT}_{\lim }-1 \mathrm{~K}$.

Given the strong sensitivity of BTs to atmospheric profiles of water vapor and hydrometeors, we also perform a series of sensitivity experiments that turn off the updates of either water-related or non-water-related state variables throughout the EnKF all-sky BT assimilation cycles. In these sensitivity experiments, we assimilated all-sky BTs and HPI every hour for $6 \mathrm{~h}$ without updating atmospheric water vapor and hydrometeors (hereafter referred to as QOFF) or updating atmospheric water vapor and hydrometeors only (hereafter referred to as QONLY). Finally, we swap the initial conditions of atmospheric water vapor and hydrometeors (referred to as Q) and other variables (wind, temperature, dry air mass, and pressure; hereafter referred to as vortex initial conditions or V) among CNTL, QOFF, and QONLY. We also perform deterministic forecasts from each of these sensitivity experiments to examine the relative importance of initial moisture and hydrometeors profiles and dynamical vortex core on RI onset. For example, a swapping sensitivity experiment which uses the vortex initial conditions from CNTL and moisture initial condition from QOFF is referred to as VcntlQqoff. The list of swapping experiment is summarized in Table 1. Since environmental conditions are replaced with the FNL analysis for all the experiments, CNTL, QOFF, and QONLY mostly differ within the circle of radius $600 \mathrm{~km}$.

\section{Analyses with and without assimilation of all-sky radiance from Himawari-8}

We first compare the hourly EnKF analyses between the three observing system experiments (OSEs), that is, assimilation of all-sky BTs from channel 8 of Himawari-8 $(\mathrm{BT}+\mathrm{HPI})$, clear-sky only BTs $(\mathrm{clrBT}+\mathrm{HPI})$, and without radiance assimilation (HPI), all of which assimilate hourly interpolated ADT best track intensity

TABLE 1. Summary of swapping sensitivity experiments discussed in section 4b.

\begin{tabular}{lcl}
\hline \hline \multicolumn{1}{c}{ Expt name } & $\begin{array}{c}\text { Vortex initialization (all state variables } \\
\text { but moisture and hydrometeors) }\end{array}$ & \multicolumn{1}{c}{$\begin{array}{c}\text { Moisture initialization (all water species including } \\
\text { moisture and hydrometeors) }\end{array}$} \\
\hline VqoffQcntl & As in QOFF & As in CNTL \\
VcntlQqoff & As in CNTL & As in QOFF \\
VqonlyQcntl & As in QONLY & As in CNTL \\
VcntlQqonly & As in CNTL & As in QONLY \\
VcntlQqoff+cntl_awn0 & As in CNTL & QOFF + azimuthal wavenumber 0 of (CNTL - QOFF) \\
\hline
\end{tabular}


(a) Observation

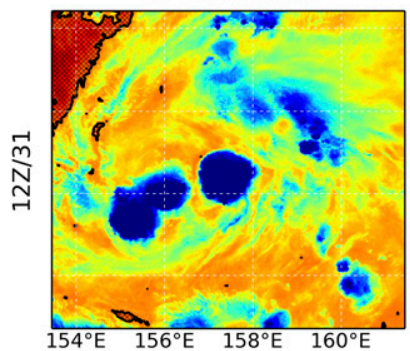

(e)

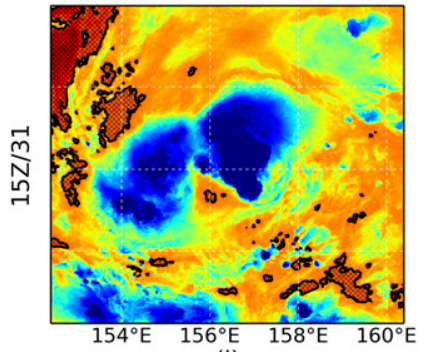

(i)

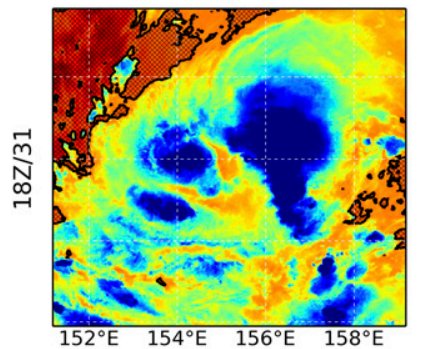

(b) EnKF analysis: $\mathrm{BT}+\mathrm{HPI}$

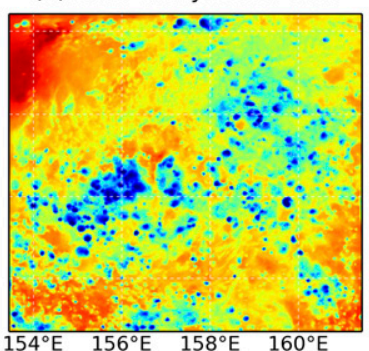

(f)

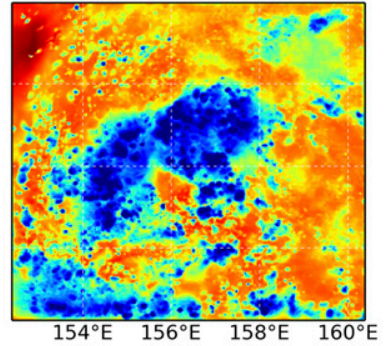

(j)

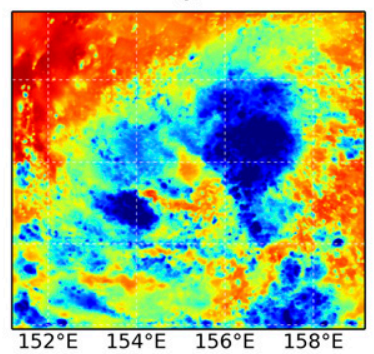

(c) EnKF analysis: clrBT+HPI

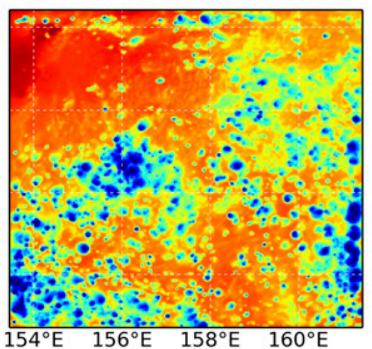

(g)

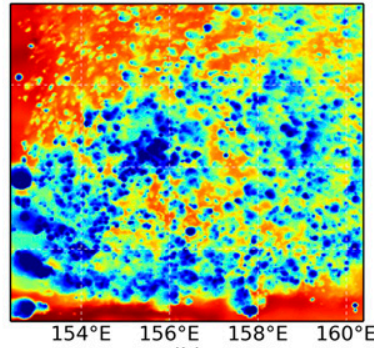

(k)

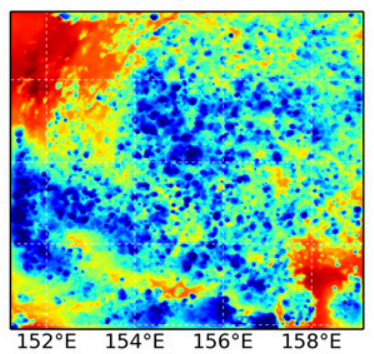

(d) EnKF analysis: HPI

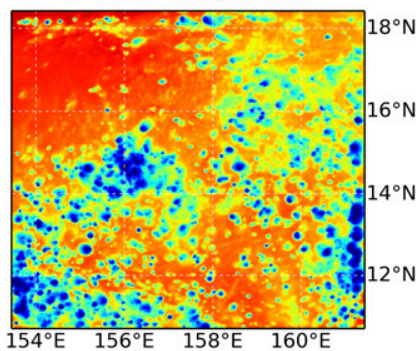

(h)

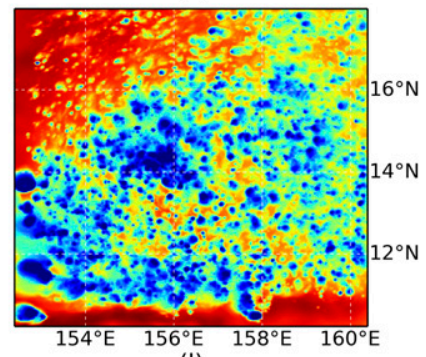

(I)

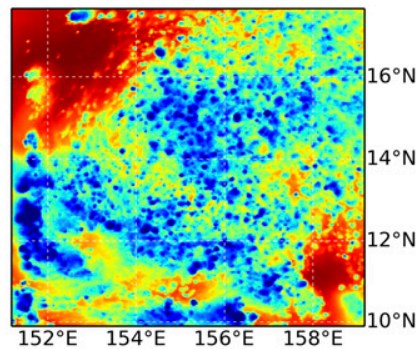

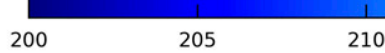

210

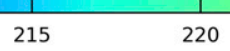

225

230

235

240

245

FIG. 3. Simulated brightness temperatures (color shading) of Himawari-8 AHI channel 8 at (a)-(d) 1200 UTC (initial assimilation cycle), (e)-(h) 1500 UTC (3-h assimilation cycle), and (i)-(l) 1800 UTC 31 Jul (6-h assimilation cycle) from the (from left to right) observation, BT + HPI, clrBT + HPI, and HPI experiments. The clear-sky observations that are assimilated in clrBT + HPI experiment are hatched in (a), (e), and (i).

in terms of minimum SLP at the observed location. Figure 3 shows the observed and simulated BTs from the 3 -km domain for channel 8 after 0,3 , and $6 \mathrm{~h}$ assimilation cycling (verified at 1200, 1500, and 1800 UTC 31 July, respectively). The observed clear-sky BTs are hatched with crossed diagonals (Fig. 3, left column). As expected, the finest $3-\mathrm{km}$ domain that focuses on innercore structure of TCs is mostly covered by clouds. The number of BT observations assimilated in the clrBT + HPI experiment is reduced from the BT + HPI experiment roughly by a factor of 10 . After the first EnKF analysis update, BT + HPI has already shown improvement over the HPI experiment in better representing high values of BTs in the northwest quadrant of the domain, and high values of BTs in the southwest corner of the domain in comparison to the observations (Fig. 3, top row). Assimilation of BTs from the water vapor-sensitive channel 8 contributes to effectively drying the originally moist northwest quadrant, and removing spuriously simulated clouds in the southwest and southeast quadrants. The clrBT $+\mathrm{HPI}$ experiment is able to capture the high BT values in the northwest corner of the domain where clear sky is observed, but the overall spatial pattern of BTs is close to HPI. Continuous hourly assimilation of all-sky BTs for 3 and $6 \mathrm{~h}$ is able to constrain the clear-sky (both dry and moist atmosphere) and convective regions at the observed locations. Conversely, both clrBT + HPI and HPI widely spread out the convection across the entire domain except for the northwest corner (Fig. 3, second and third rows). Figure 4 compares the BTs of channel 14 (wavelength is $11.2 \mu \mathrm{m}$ ), which is not assimilated but sensitive to particulates (i.e., cloud and land surface). The observed BTs in the convective regions that reach 
(a) Observation

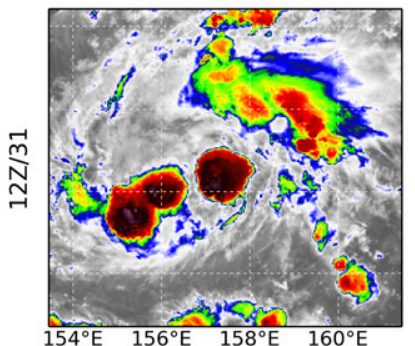

(e)

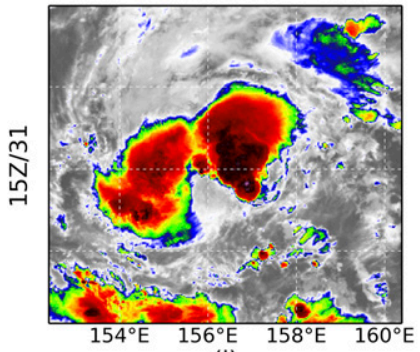

(i)

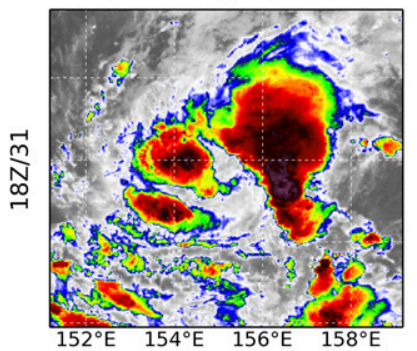

(b) EnKF analysis: $\mathrm{BT}+\mathrm{HPI}$

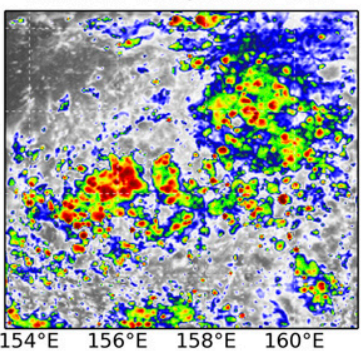

(f)

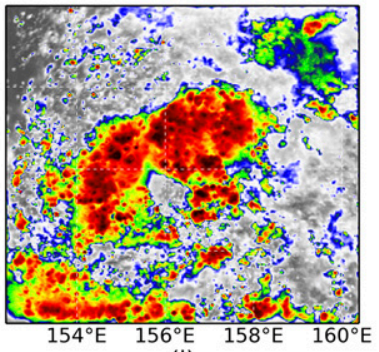

(j)

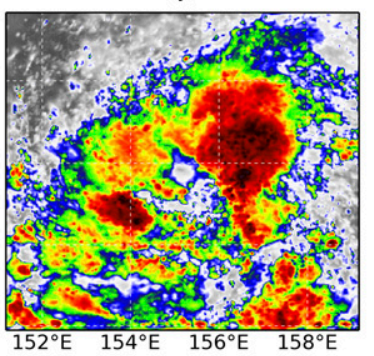

(c) EnKF analysis: clrBT+HPI

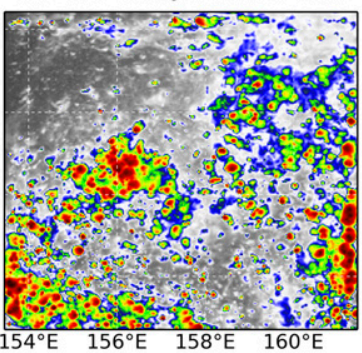

(g)

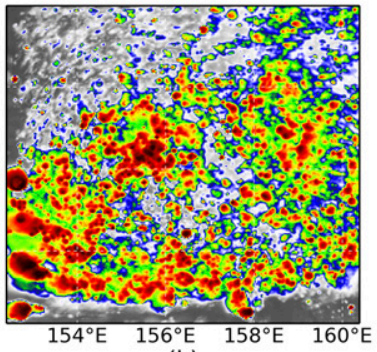

(k)

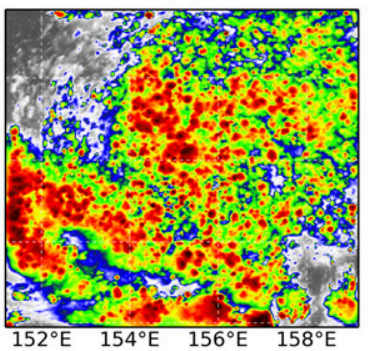

(d) EnKF analysis: HPI

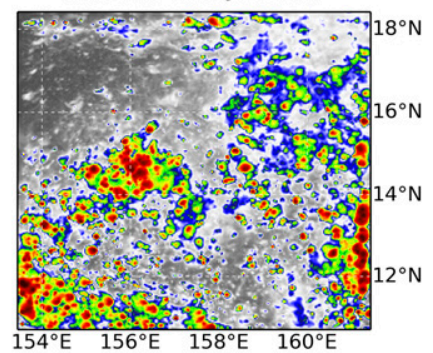

(h)

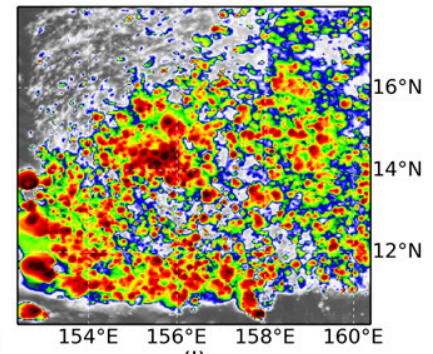

(I)

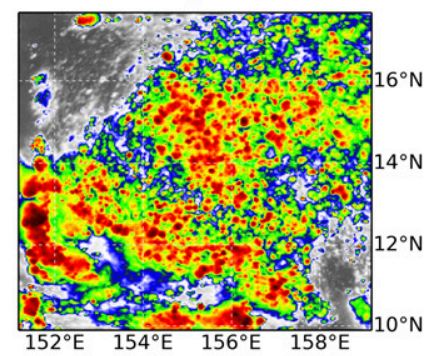

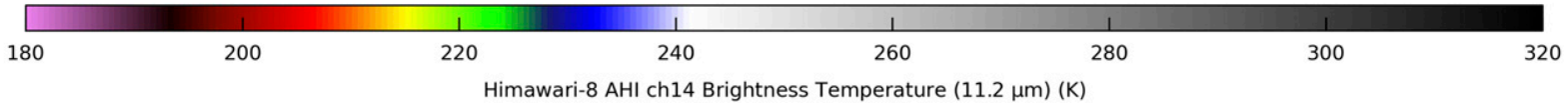

FIG. 4. As in Fig. 3, but for channel 14.

as low as $195 \mathrm{~K}$ at $1800 \mathrm{UTC}$ are well represented by BT+HPI. Meanwhile, the analyzed BTs in channel 14 from clrBT + HPI and HPI are broadly covered by low clouds with considerably higher BTs. Not only does BT +HPI capture the observed clear- and cloudy-sky distribution, but it also forms comparable high clouds.

The impacts of assimilating all-sky BTs on TC innercore structures are exhibited in Fig. 5 by comparing the temporal evolution of EnKF background, analysis, and analysis increments. The dry initial inner core, in particular, from the middle to upper troposphere, is effectively moistened by the first data assimilation cycle (Fig. 5, top row), and further develops into a moist innercore vortex through the continuous 6-hourly cycles (Fig. 5, second and third rows). Along with moistening of the inner-core region, assimilation of all-sky BTs also contributes to strengthening of the TC vortex. Continuous hourly cycling enables the formation of a moist strong inner-core vortex, which is necessary for the development of TCs. Although the mid- to lower-tropospheric moisture is not directly sensitive to BT calculations under the existence of overwhelming clouds while the wind field is not directly used in the BT calculation, Fig. 5 shows that the flow-dependent ensemble-based covariances can update the inner-core moisture and vortex structure in a dynamically consistent manner, as shown by Fig. 1 of ZMC16.

We further examine the impacts of assimilating BTs on the forecasts ${ }^{2}$ of TC intensity and structure. Figure 6 compares the intensity forecasts in terms of minimum

\footnotetext{
${ }^{2}$ Strictly speaking this is not a forecast but a simulation since the lateral boundary conditions are derived from the GFS FNL analysis, which is designed to isolate the impact of initial conditions in the forecast. Given the large domain 1 used for WRF (Fig. 1), the use of FNL analysis does not significantly affect the quality of the WRF forecast of Soudelor, at least not for the first 3 days (not shown).
} 
(a) EnKF background

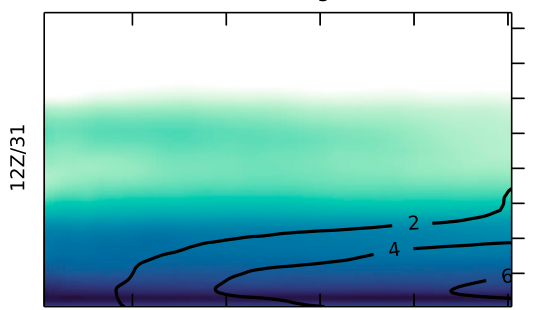

(d)

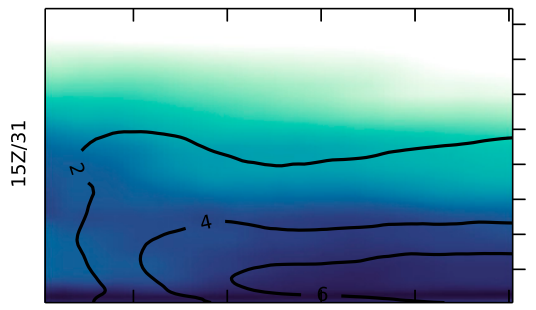

(g)
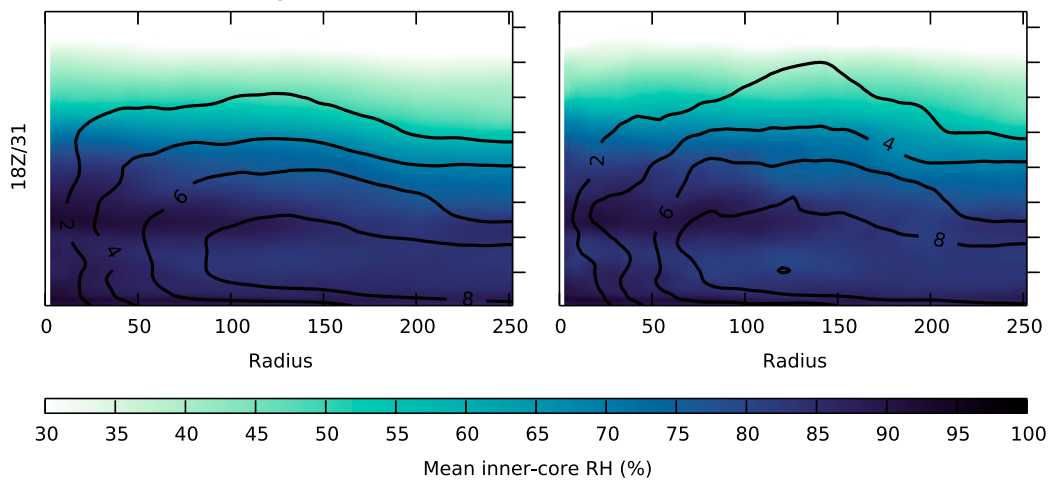

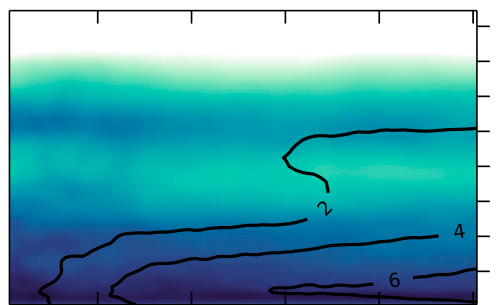

(e)

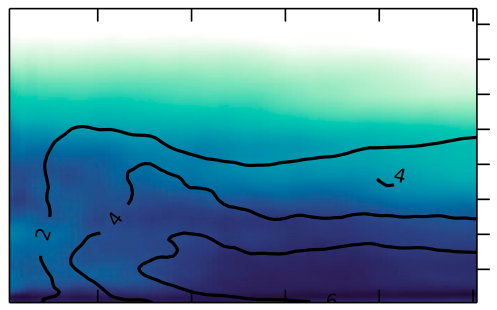

(h)

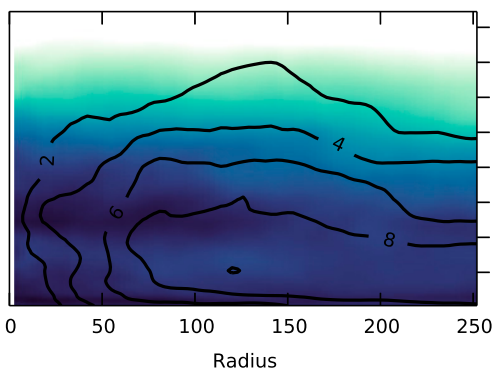

(b) EnKF analysis (c) Analysis increment

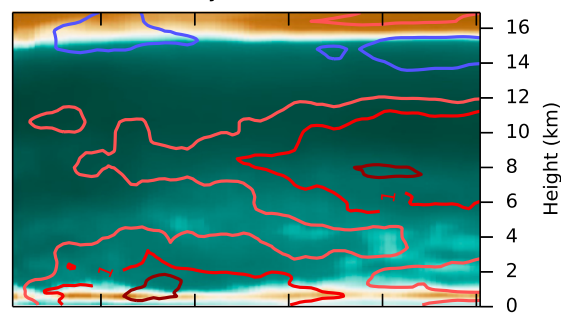

(f)

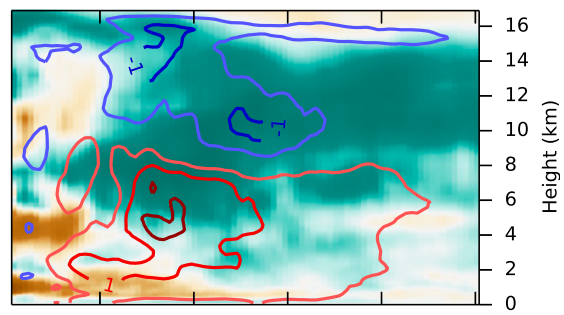

(i)

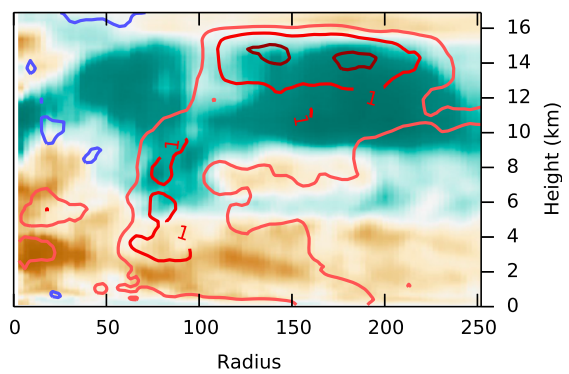

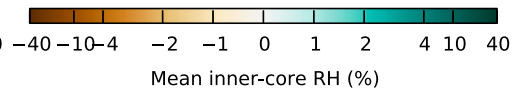

Mean inner-core $\mathrm{RH}(\%)$

FIG. 5. Azimuthally averaged inner-core relative humidity (color shaded) and tangential wind (contour; $\mathrm{m} \mathrm{s}^{-1}$ ) of (a),(d),(g) EnKF background; (b),(e),(h) EnKF analysis; and (c),(f),(i) EnKF analysis increment.

SLP and maximum 10-m wind speed between deterministic forecasts initialized from different EnKF mean analyses. Also plotted are the deterministic forecasts from the EnKF prior mean at initial assimilation time (which did not assimilate any observations, hereafter referred to as NoDA), and two sets of best track observational intensity estimates (one provided by University of Wisconsin-Madison using ADT technique and the second by the JTWC) for comparison and reference. All deterministic forecasts from BT + HPI experiments predict the rapid deepening of Soudelor, which verified reasonably well with the storm's observed intensification rate indicated by the two best track estimates. On the contrary, none of the deterministic forecasts from clrBT + HPI, HPI, or NoDA is able to capture the RI timing and intensification rate of Soudelor, although the assimilations of HPI and clear-sky BTs slightly improve the forecasts over NoDA. The favorable environment of Soudelor still allows the clrBT + HPI, HPI, and NoDA to simulate an intensifying vortex, but their RIs are largely delayed (or absent) compared to
$\mathrm{BT}+\mathrm{HPI}$ and best track datasets by approximately 24-48 h. The departure of BT+HPI from clrBT $+\mathrm{HPI}$, HPI, and NoDA clearly demonstrates the positive contribution of assimilating all-sky infrared BTs from a geostationary satellite to the prediction of rapidly intensifying TCs, consistent with our previous studies (ZMC16; Minamide and Zhang 2017).

The positive impacts of all-sky BT assimilation on the analyses and forecasts of the tropical cyclone structure in terms of both dynamic and thermodynamic fields are further examined. Figure 7 examines the temporal evolution of the simulated maximum radar reflectivity, low-pass filtered ${ }^{3}$ potential vorticity at the lower and upper troposphere of the deterministic forecasts from

\footnotetext{
${ }^{3}$ Here, we conduct 2D Fourier decomposition on the horizontally detrended potential vorticity fields to divide the original fields into scales with 2D horizontal wavelengths larger than $200 \mathrm{~km}$. This is designed to remove the small-scale structures and to highlight the vortex-scale flow.
} 
(a)

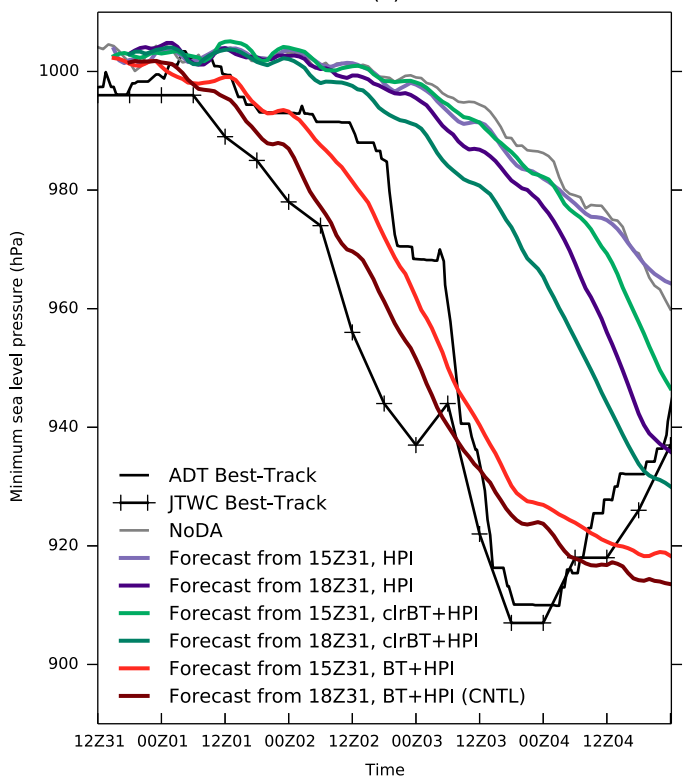

(b)

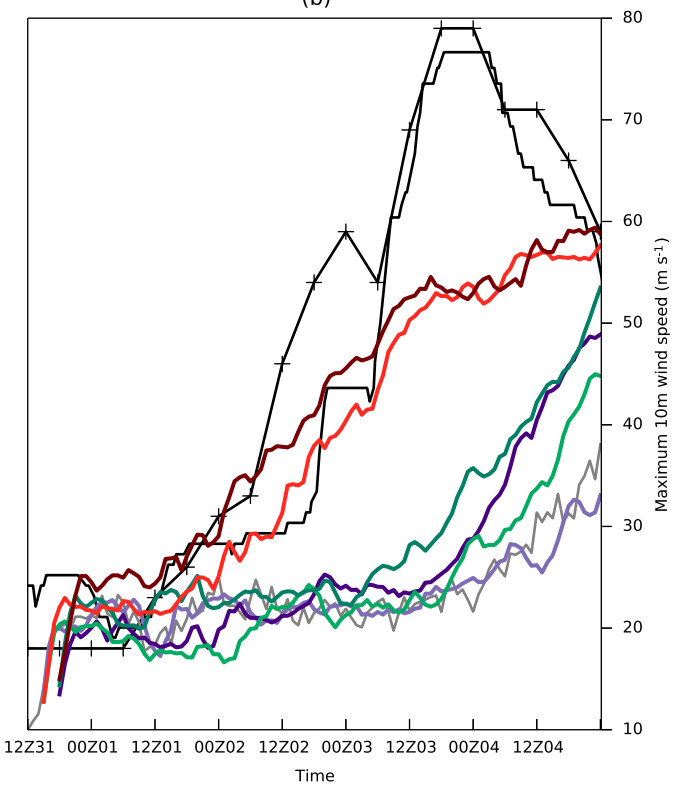

FIG. 6. Time evolution of tropical cyclone intensity in terms of (a) minimum sea level pressure (hPa) and (b) maximum 10-m wind speed $\left(\mathrm{m} \mathrm{s}^{-1}\right)$ for the best track datasets and different EnKF experiments forecasts (color coded). Note that in (b), the best track represents the 1-min sustained maximum wind speed, while instantaneous maximum wind speed is shown for each experiment. The analyses in the main text are based on minimum SLP, which is consistently comparable among experiments and best track datasets, but here we also show the wind speed for reference purposes.

1800 UTC 31 July. Experiment BT+HPI produces a stronger TC vortex than both clrBT + HPI and HPI in both the lower and upper troposphere from the very beginning, along with stronger associated convective activity (Figs. 7a-c). Snapshots of the 1.5-, 9-, and 36-h forecasts illustrate that BT+HPI is able to sustain the stronger vortex and associated convection than clrBT + HPI and HPI at all forecast times. The stronger convection in BT + HPI leads to larger latent heating, which facilitates a faster-developing TC vortex (Fig. 7, first column). In comparison, an initially weaker TC vortex with initially weaker convection, as in clrBT+HPI and HPI, is not able to strengthen without sustained convective activity (Fig. 7, second and third columns). The 36-h forecast from clrBT +HPI becomes slightly stronger than HPI, potentially due to better analyzed clear-sky fields but is still considerably weaker than BT+HPI. Note that at the analysis time (Fig. 7, top row), most of the domain 3 is covered by the light-to-moderate precipitation because it represents the ensemble mean of bounded positive-definite hydrometeors fields. This unrealistic widespread precipitation soon diminishes after only $1.5 \mathrm{~h}$ of simulation (Fig. 7). The comparison of infrared BTs (Figs. 3 and 4) and radar reflectivity (Fig. 7) suggests that our assimilation of all-sky BTs may not adequately constrain the precipitation-liquid, -snow, and -graupel variables. This is likely because infrared BTs are more sensitive to top of the cloud than the precipitating hydrometers below. These hydrometers are expected to be further improved by simultaneously assimilating more precipitation-sensitive observations such as microwave satellite radiances in the future.

In short summary, comparison of BT $+\mathrm{HPI}$, clrBT $+\mathrm{HPI}$, and HPI indicates that assimilation of all-sky BTs not only helps to improve the thermodynamic variables such as temperature and moisture-to which satellite BTs are directly sensitive-but also helps to update dynamical state variables such as the TC vortex initial conditions, and thus improve subsequent forecasts. The relative significance of the moisture versus initial vortex updates in Soudelor's rapid intensification is examined in the following section.

\section{Impacts of moisture and hydrometer updates on tropical cyclone initialization}

\section{a. Sensitivity experiments to updating moisture in the EnKF analysis}

To further examine the impacts of assimilating BTs on analyzing the atmospheric moisture and hydrometeors, two additional data assimilation sensitivity experiments are performed. These experiments are identical to BT+HPI except that either all water-related or all nonwater-related model state variables are not updated during data assimilation. The first experiment, QOFF, does 
(a) $\mathrm{BT}+\mathrm{HPI}$

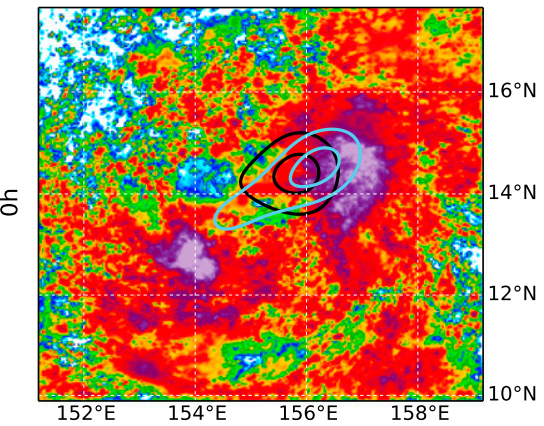

(d)

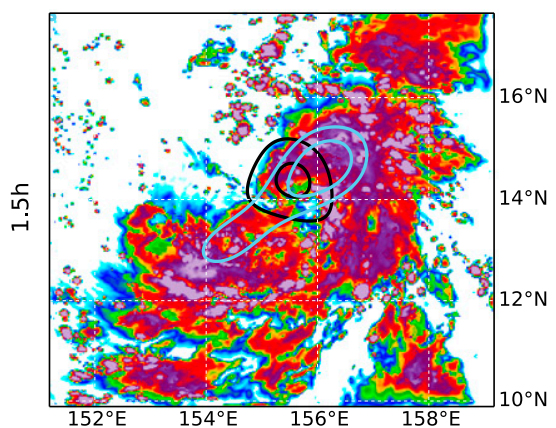

(g)

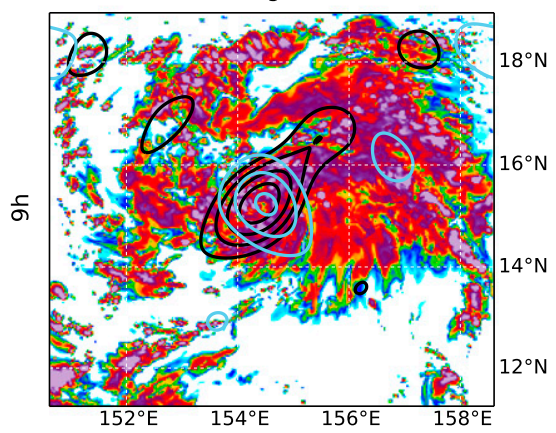

(j)

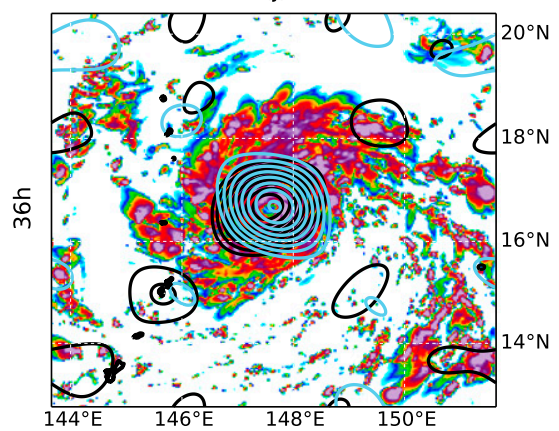

(b) $\mathrm{CIrBT}+\mathrm{HPI}$

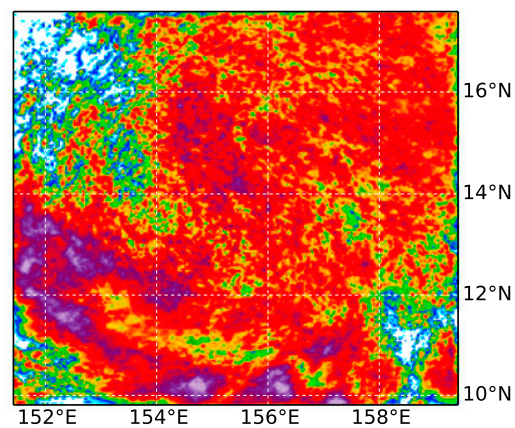

(e)

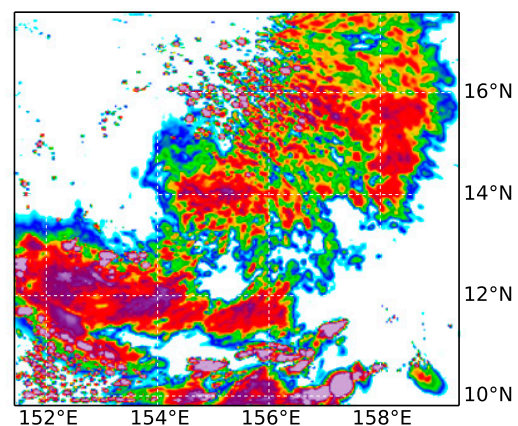

(h)

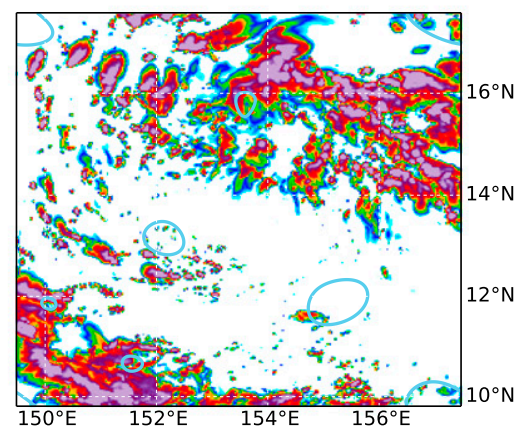

(k)

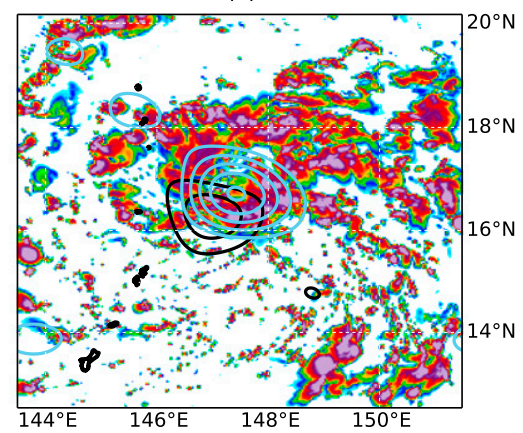

(c) HPI

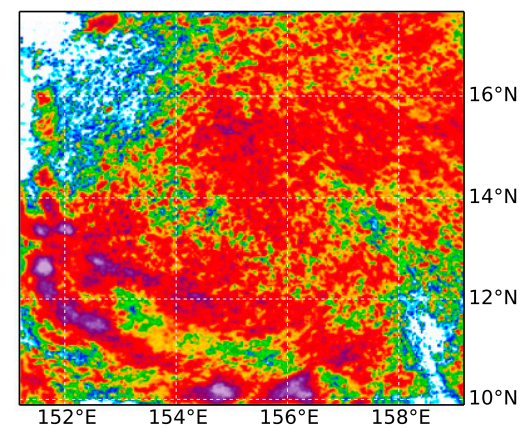

(f)

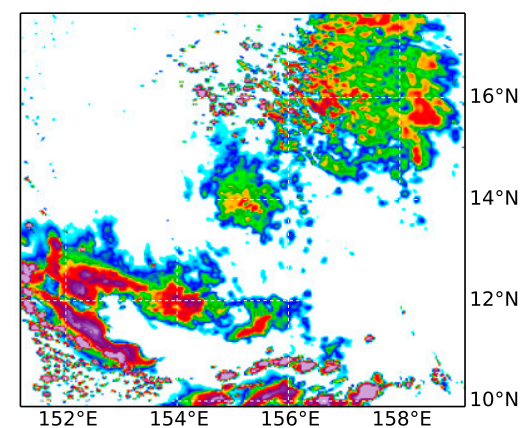

(i)

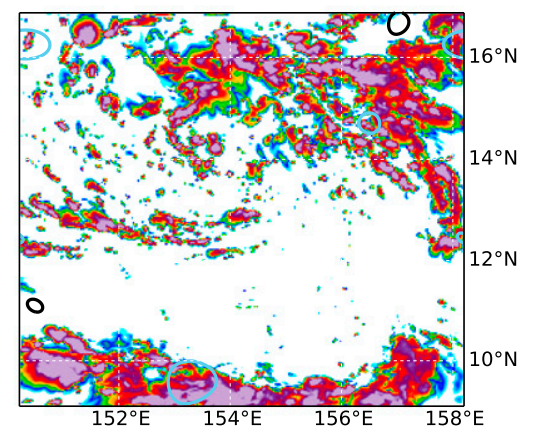

(I)

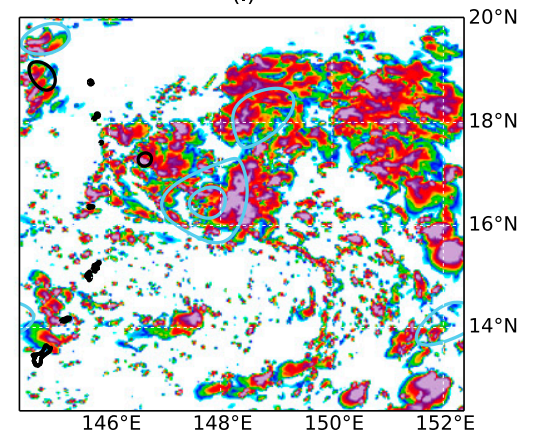

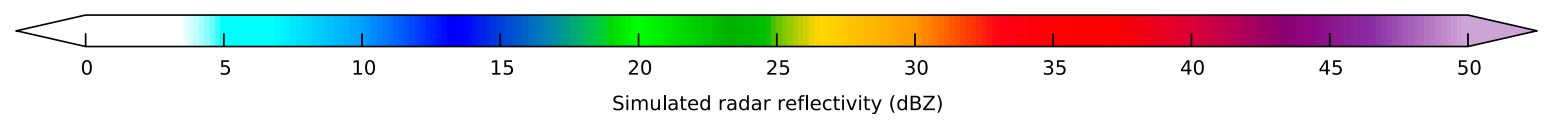

FIG. 7. Simulated radar reflectivity (color shading) and low-pass filtered ( $>200 \mathrm{~km}$ ) potential vorticity at $850 \mathrm{hPa}$ (black contours) and at $400 \mathrm{hPa}$ (sky-blue contours) for the deterministic forecasts at (a)-(c) 1800 UTC 31 Jul 2015 (initialization time of forecast, which is after $6 \mathrm{~h}$ of data assimilation), and the (d)-(f) 1.5-, (g)-(i) 9-, and (j)-(l) 36-h forecasts, from the (left) BT+HPI, (middle) clrBT+HPI, and (right) HPI experiments. Note that the unnatural wavelike patterns in some vorticity fields show up because of the Fourier decomposition process in low-pass filtering. 
(a) EnKF analysis: QOFF
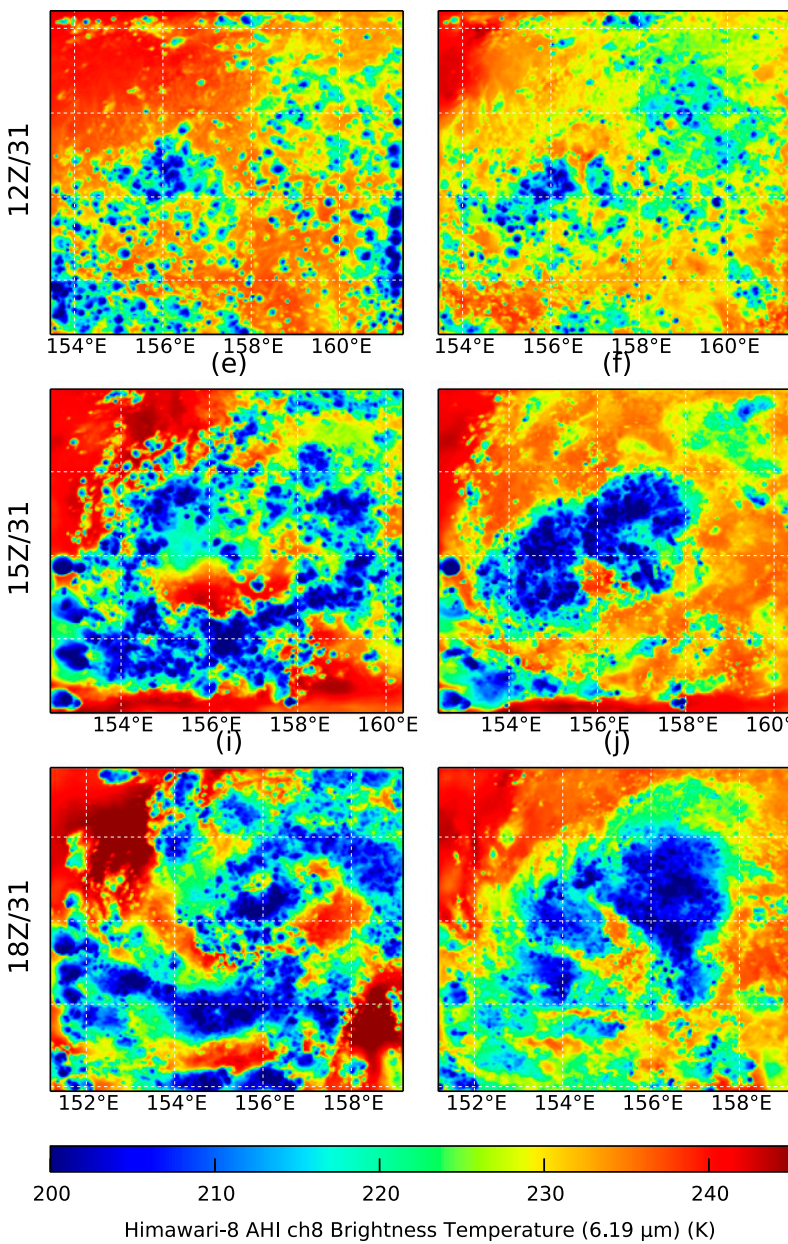

(c) EnKF analysis: QOFF
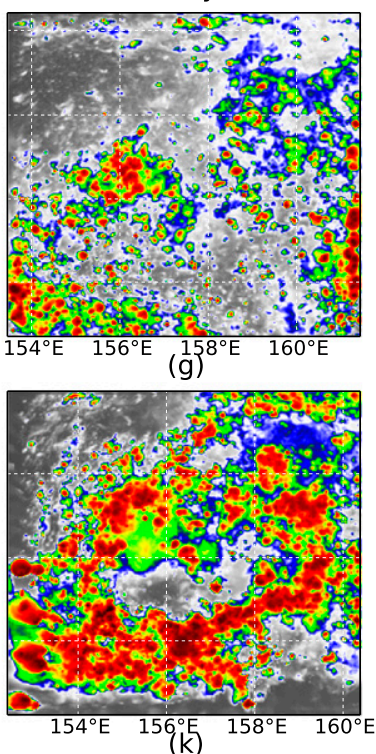

$(\mathrm{k})$
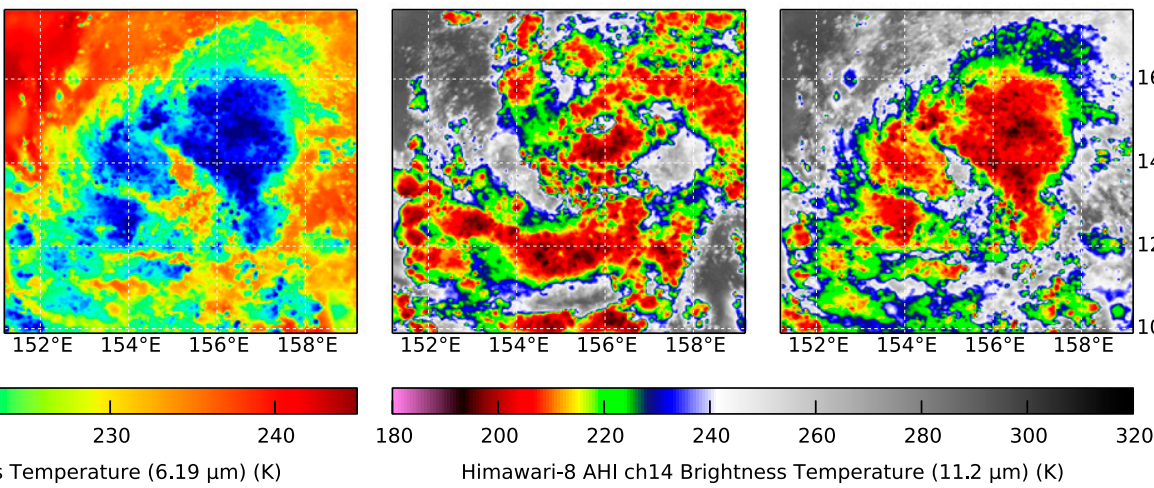

(d) EnKF analysis: QONLY
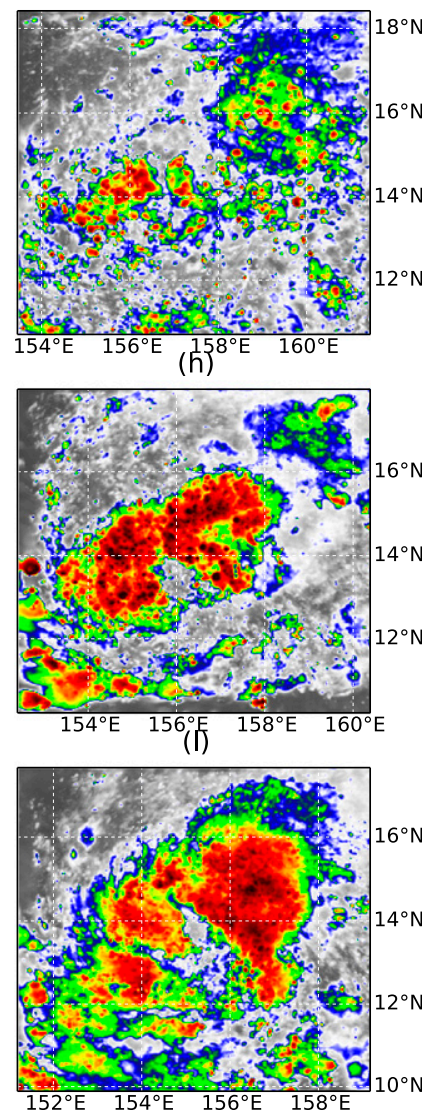

20

FIG. 8. (two left columns) As in Fig. 3, but for the (a),(e),(i) QOFF and (b),(f),(j) QONLY experiments, and (two right columns) as in Fig. 4, but for the (c),(g),(k) QOFF and (d),(h),(l) QONLY experiments.

not update the water vapor or any of the liquid and solid hydrometeors in each of the hourly EnKF analysis cycles (i.e., no water, cloud, or precipitating hydrometeors are updated at all). The second experiment, QONLY, turns off the EnKF analysis updates for all nonwater model state variables (i.e., only water-related state variables are updated). These two experiments are uniquely designed to explore the importance of initial inner-core moisture analysis in the tropical cyclone prediction as highlighted in the recent study of Emanuel and Zhang (2017).

Comparisons of QOFF and QONLY to the control experiment BT+HPI (with full 6-hourly EnKF analysis cycles; referred to as CNTL) in terms of simulated BTs of Himawari-8 AHI channels 8 and 14 from the EnKF analyses, analyzed azimuthal mean inner-core structures, forecasted TC intensities, radar reflectivity, and filtered potential vorticity are shown in Figs. 8-11. Without the EnKF updates of any water species, the analyzed convective activity in QOFF resembles that in HPI. Both
QOFF and HPI simulate a spurious convective line along $12^{\circ} \mathrm{N}$, while both fail to model enhanced convective activity around $156.5^{\circ} \mathrm{N}$ and $14.5^{\circ} \mathrm{N}$ at 1800 UTC July 31 (Fig. 8, first and third columns; also see Figs. 3 and 4). Although updating dynamic variables does help formation of a strong vortex, similar in strength to CNTL, at 1800 UTC in the QOFF experiment, the lack of moisture update in QOFF results in the TC inner-core being much drier than CNTL and more comparable to HPI (Fig. 9). The different representation of convective activity in QOFF, together with subsequent differences in the modeled initial tropical cyclone vortex, eventually leads to a delay in the timing of rapid intensification (RI) and an overall weaker intensity than in CNTL (light blue vs dark red lines in Figs. 10a and 10c). More specifically, QOFF lacks convective activity surrounding the TC vortex (Fig. 11, first column), which likely contributes to QOFF being more vulnerable to environmental influences such as vertical wind shear. After $9 \mathrm{~h}$ of integration, the TC vortex 
(a) QOFF - CNTL

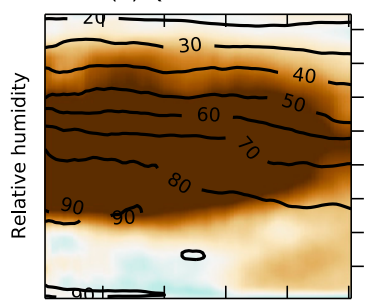

(e)

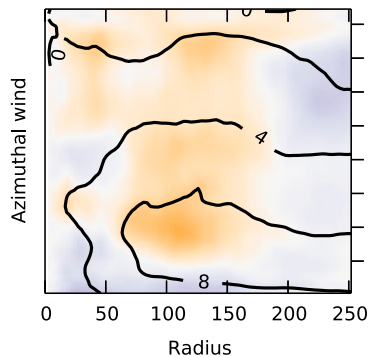

(b) QOFF - HPI

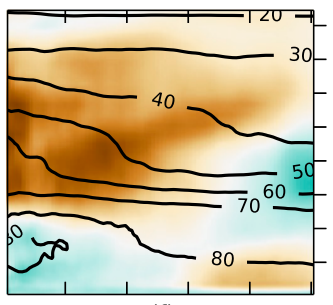

(f)

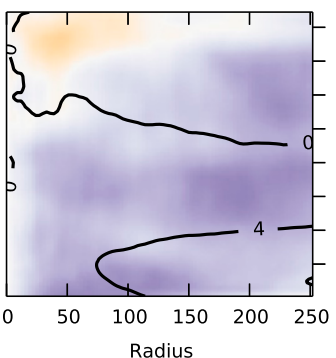

(c) QONLY - CNTL

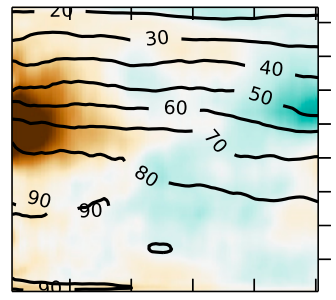

(g)

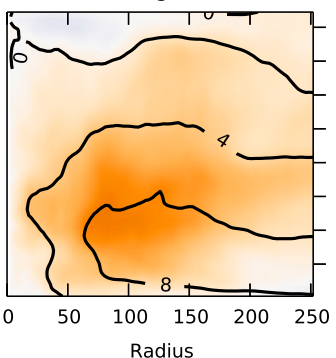

(d) QONLY - HPI

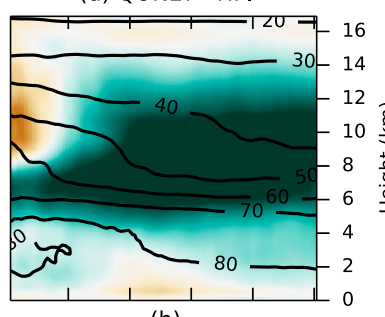

(h)

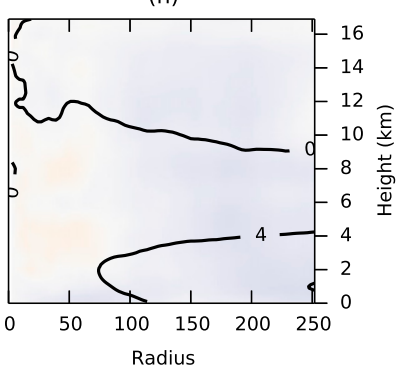

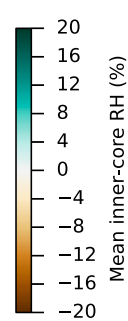

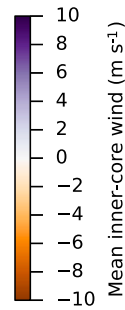

FIG. 9. Inner-core EnKF analysis (color shaded) of (a)-(d) relative humidity and (e)-(h) azimuthal wind. Color shades are (from left to right) QOFF - CNTL, QOFF - HPI, QONLY - CNTL, and QONLY - HPI at 1800 UTC 31 Jul 2015. Contours are from CNTL in (a), (c), (e), and (g) and from HPI in (b), (d), (f), and (h).

in QOFF has a larger tilt than that in CNTL (approximately $200 \mathrm{vs} 50 \mathrm{~km}$ ), which leads to a delayed development. Thus, insertion of water vapor and hydrometeors through the EnKF assimilation of all-sky radiances helps to moisten the atmosphere. This can modify the distribution and strength of convective activity, and subsequently the structure and intensity of the tropical cyclone in both the analysis and forecast. (a) QOFF

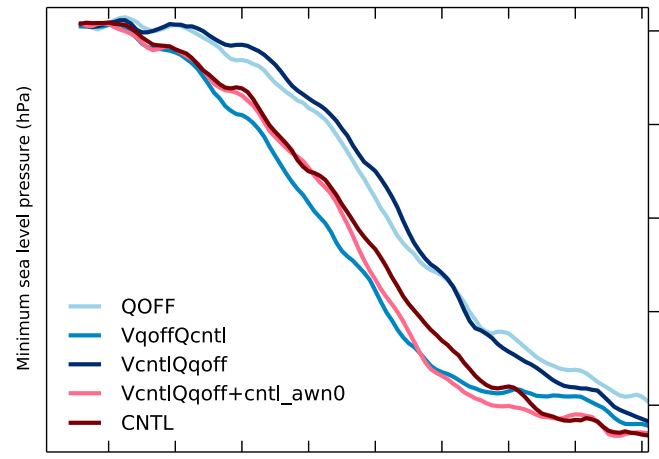

(c)

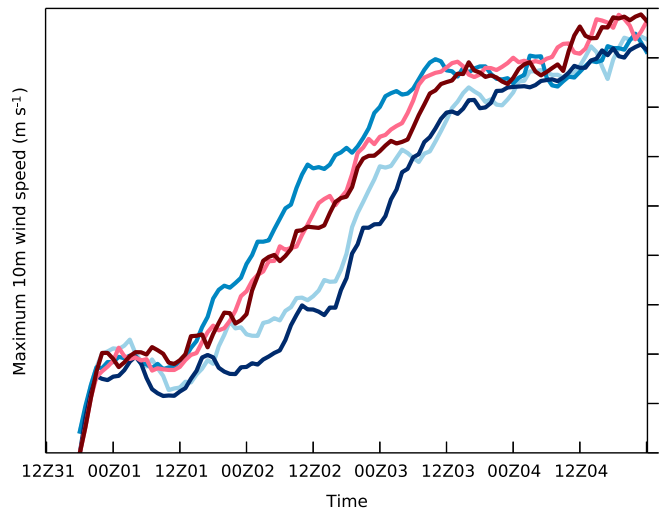

(b) QONLY

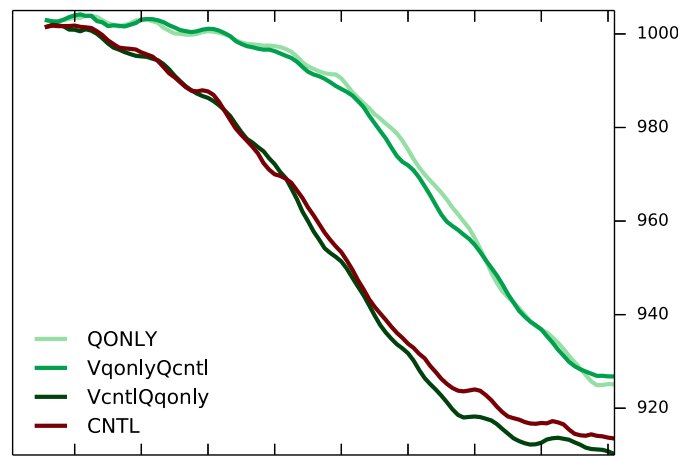

(d)

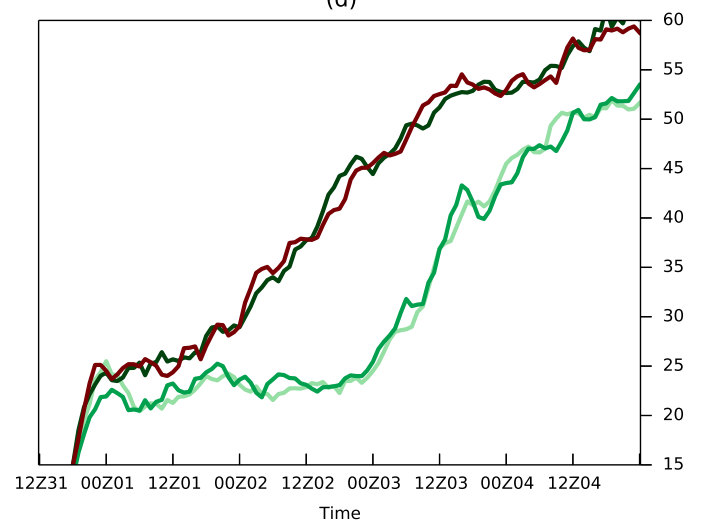

FIG. 10. As in Fig. 6, but for (a),(c) QOFF, VqoffQcntl, VcntlQqoff, and VcntlQqoff+cntl_awn0, and (b),(d) QONLY, VqonlyQcntl, and VentlQqonly. 
(a) QOFF

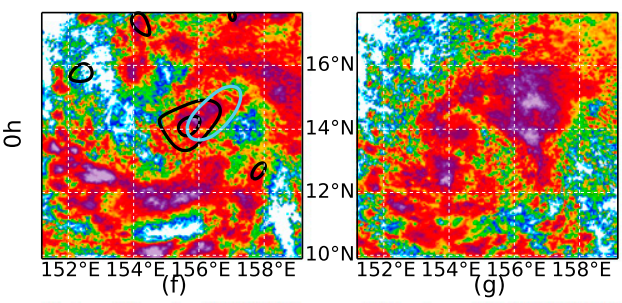

(c) VqoffQcntl

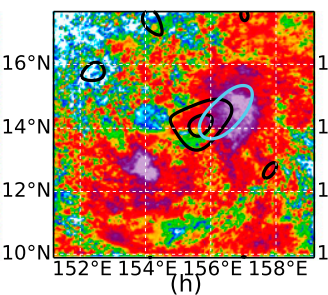

(d) VentiQqoff

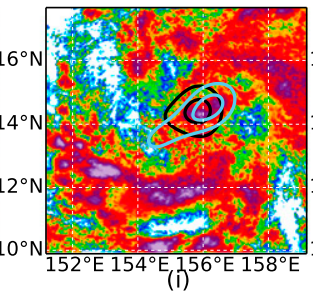

(e) VcntlQqoff+cntl_awno

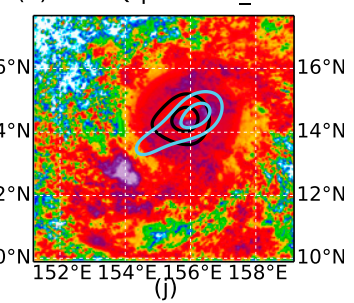

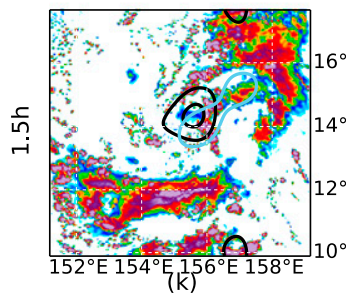
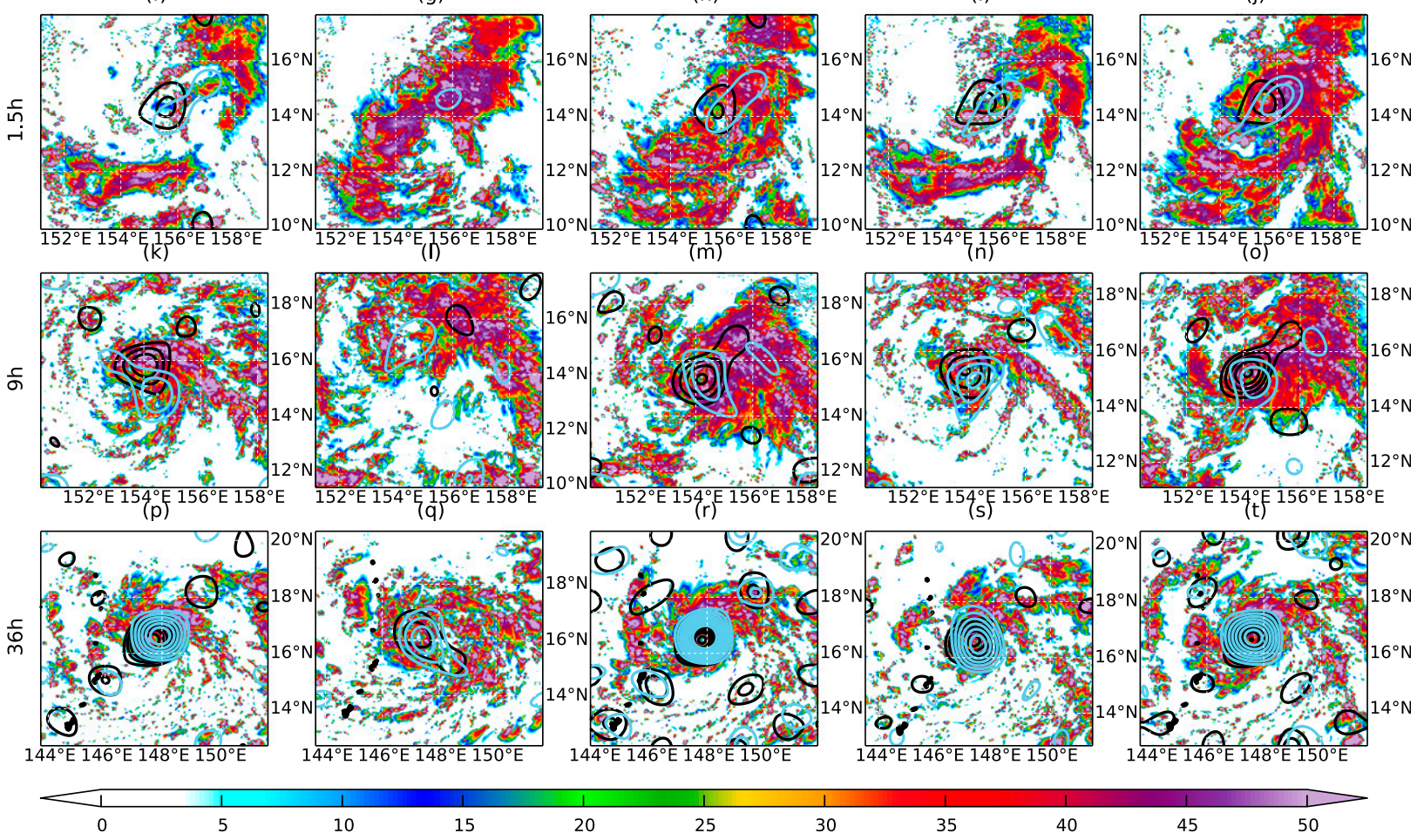

10

15

$\begin{array}{ccc}1 & 1 & 1 \\ 20 & 25 & 30 \\ \text { Simulated radar reflectivity } & (\mathrm{dBZ})\end{array}$

35

40

45

50

FIG. 11. As in Fig. 7, but for (a),(f),(k),(p) QOFF; (b),(g),(l),(q) QONLY; (c),(h),(m),(r) VqoffQcntl; (d),(i),(n),(s) VcntlQqoff; and (e),(j),(o),(t) VcntlQqoff+cntl_awn0.

Even with only the water vapor updates during the EnKF analysis, QONLY well captures the observed pattern of clear-/cloudy-sky distribution (Fig. 8, second and fourth columns; see also Figs. 3 and 4). In Fig. 9 (third and fourth columns), QONLY's TC inner core is overall much moister than in HPI at 1800 UTC 31 July, although it still fails to analyze a moist upper troposphere within the $50-\mathrm{km}$ radius from the vortex center. Meanwhile, the modeled wind field is clearly too weak and inferior to BT+HPI. Likely resulting from the lack of a sufficiently strong primary circulation, the TC vortex of QONLY fails to capture the intensification rate of Soudelor (Figs. 10b,d). Even with the initially active convection, QONLY is not able to sustain the active convection to develop a strong vortex (Fig. 11, second column). Thus, a deficient update of either dynamic variables or water variables during the data assimilation cycles will potentially degrade the EnKF analyses and subsequent forecasts. In other words, assimilation of allsky BTs with flow-dependent error covariances is demonstrated here to have great potential for considerably improving the TC analysis and forecast through better constraining and updating not only the water vapor but also the other dynamic state variables.

\section{b. Impacts of vortex and/or moisture initialization}

To further evaluate the relative importance of vortex versus moisture initialization on Soudelor's intensification and forecasts, we perform several additional sensitivity experiments whose initial conditions are swapped among experiments CNTL, QOFF, and QONLY after the final analysis cycle of 1800 UTC July. We first import the analyses of all water-related variables (i.e., water vapor, cloud water, rainwater, ice, snow and graupel mixing ratio) from the CNTL EnKF analysis mean to replace those in QOFF but otherwise keep the 
analyses of all other state variables the same as in QOFF. This new experiment is hereafter referred to as VqoffQcntl, which is designed to examine how much is gained exclusively through updating water-related (state) variables from EnKF assimilation of all-sky BTs. The moisture and vortex initializations of swapping sensitivity experiments are also listed in Table 1. Note that the initial dynamical adjustments in these swapping experiments do not significantly differ from the corresponding original forecasts, since the adjustments are mostly dominated by the initializations of non-water-related state variables. The temporal evolutions of intensities, radar reflectivity and filtered potential vorticity for this experiment are compared in Figs. 10 and 11.

In comparison to the original QOFF EnKF experiment without updating the moisture state variables, the forecast experiment VqoffQcntl drastically improves the forecast over QOFF (Figs. 10a,c). The TC vortex in VqoffQcntl intensifies almost identically to that of CNTL for the first $24 \mathrm{~h}$. The development is slightly faster than CNTL thereafter but the tropical cyclone eventually reaches a comparable intensity with CNTL at the matured stage. In particular, as shown in Fig. 11 (first and third columns), the use of improved initial conditions in water-related variables in VqoffQcntl is able to successfully develop more enhanced and sustained deep convection than in QOFF. Both QOFF and VqoffQcntl do simulate nearly identical vortices at $1.5 \mathrm{~h}$ of the forecast, but more enhanced convection enables VqoffQcntl to develop a stronger and less-tilted vortex than QOFF at subsequent forecast times, which eventually leads to earlier development and intensification of Soudelor.

Conversely, we replace all the nonwater variables (i.e., wind velocity, pressure, temperature, dry air mass, and geopotential) in QOFF with those from CNTL while retaining the analyses of all water vapor and hydrometeors (or the lack of the update) as a new experiment, VcntlQqoff (Table 1). Although initialized with the same vortex as CNTL, the poorer moisture fields of QOFF largely degrade the forecast from VentlQqoff compared to CNTL (Figs. 10a,c). The convective activity in the inner-core regions of VcntlQqoff is slightly more enhanced than in QOFF, potentially by a better CNTL vortex that is consistent with the observed convective activity, but clearly to a much lesser degree, and with a larger vortex tilt than CNTL (Fig. 11, first and fourth columns). Likely because of less active inner-core convection, the TC vortex in VentlQqoff intensifies almost identically to QOFF, which is much weaker than CNTL. These results again indicate that updating moisture and hydrometers through the EnKF assimilation cycles helps forecasts better capture observed convective activity. Additionally, assimilation of all-sky infrared radiances can help develop a more resilient and stronger TC vortex that facilitates subsequent development and rapid intensification.

Given the inability for experiment VcntlQqoff to reach the same maximum intensity as in CNTL, we further modify the moisture initialization in VentlQqoff to be closer to that in CNTL. More specifically, we add the azimuthal wavenumber- 0 structure of the moisture difference between CNTL and QOFF to VcntlQqoff (hereafter referred to as VcntlQqoff + cntl_awn0; Table 1) to examine whether the representation of azimuthal wavenumber0 component of moisture is sufficient to predict the RI process as forecasted in CNTL. In other words, CNTL and VcntlQqoff+cntl_awn0 only differ in moisture and hydrometeor variables in wavenumbers other than 0 . In this case, the moisture structures of CNTL and QOFF mainly differ in the midtroposphere and boundary layer, whose structures are dominated by the axisymmetric component (not shown). This experiment is designed following Emanuel and Zhang's (2017) study, which showed that the large sensitivity of TC intensity on fully asymmetric perturbed moisture initializations with the WRF model can be comparably reproduced by perturbing initial moisture conditions with an axisymmetric TC model. Indeed, as shown in Figs. 10a and 10c, VcntlQqoff + cntl_awn0 intensifies very comparably with CNTL, indicating the dominant contribution of azimuthal wavenumber-0 moisture component to capture the RI onset in this case. We have also performed additional experiments that add the azimuthal wavenumber- 1 and -2 components of initial moisture differences in addition to azimuthal wavenumber- 0 component, but their results do not significantly differ from VcntlQqoff + cntl_awn0 (not shown). These sensitivity experiments highlight the large impacts of wavenumber- 0 midtroposphere and boundary layer moisture on TC prediction, at least at this stage of Soudelor.

Figure 12 compares the temporal evolution of the vortex tilt (i.e., the distance between the center of the filtered PV fields at 400 and $850 \mathrm{hPa}$ ) and the deep-layer vertical wind shear magnitude between $300-$ and $600-\mathrm{km}$ radius [the choices of the vertical layers and horizontal extent for evaluating these quantities is analogous to past studies of Zhang and Tao (2013), Tao and Zhang (2014, 2015), and Munsell et al. (2017)]. In first 6h, the vortex tilt of QOFF and VentlQqoff starts to deviate from CNTL, VqoffQcntl, and VentlQqoff+cntl_awn0, although their vertical wind shear magnitudes are similar in first $6 \mathrm{~h}$. After $12 \mathrm{~h}$, the vertical wind shear magnitudes of QOFF and VentlQqoff also become larger than the other experiments, as part of their tilted vortices themselves are included in the calculation 

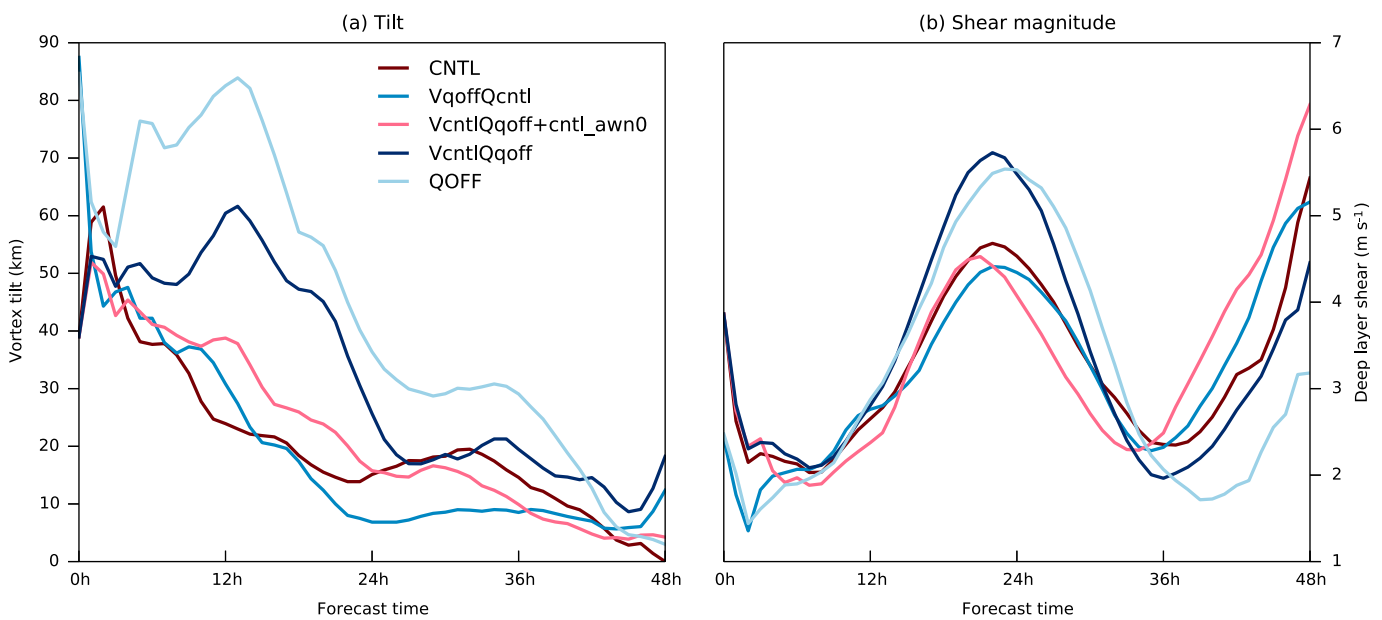

FIG. 12. Time evolution of (a) vortex tilt and (b) deep-layer shear magnitude, color-coded for each experiment as shown in the figure. Vortex tilts are computed as the distance between the vortex centers at 400 and $850 \mathrm{hPa}$. Deeplayer shear magnitudes are the mean vertical wind shear between 200 and $850 \mathrm{hPa}$ at 300 - to $600-\mathrm{km}$ radii from the vortex center. All vortex centers are the maximum grid point of low-pass filtered potential vorticity field whose scales of smaller than $200 \mathrm{~km}$ is cut by two-dimensional Fourier decomposition.

of near-TC environmental vertical wind shear. Thus, it is suggested that the lack of convection in QOFF and VcntlQqoff leads to the vortex being more vulnerable to environmental conditions, and more slanted by the surrounding vertical wind shear. The alignment of QOFF and VentlQqoff is largely delayed as the more tilted vortices struggle to realign.

Positive feedbacks between moist convection and a stronger vortex, through air-sea exchanges (Emanuel 1995; Zhang and Emanuel 2016), help the TC vortex to develop from the lower to upper troposphere. Figure 13 shows the vertical distribution of inner-core relative humidity, relative vorticity, and perturbation potential temperature. The inner-core structures CNTL, VqoffQcntl, and VentlQqoff+cntl_awn0 subsequently are able to develop a much stronger vortex (Fig. 13, first column). This results in approximately $24 \mathrm{~h}$ earlier formation of a warm core (Fig. 13, second column). In other words, the negative feedbacks between lack of convective heating and a more vulnerable, more expanded, and more slanted TC vortex leads to a delayed intensification of Soudelor.

We have also conducted forecast experiments VqonlyQcntl and VcntlQqonly that retain the analysis of all state variables in QONLY but replace all water species or all nonwater variables with CNTL, respectively (Table 1). As is the case for experiments VqoffQcntl and VcntlQqoff, the TC intensities forecasted by VqonlyQcntl and VentlQqoff are similar to their original initializations of nonupdated variables (Figs. 10b,d). With QONLY's well-represented moisture and poorly analyzed vortex, VqonlyQcntl intensifies the TC almost identically to original QONLY, while VcntlQqonly significantly improves the forecast over both QONLY and VqonlyQcntl and becomes almost identical to CNTL. Thus, at least in this case, the update in moisture variables are not sensitive to simultaneously updating other dynamic state variables (i.e., CNTL) or not (i.e., QONLY), and both moisture initializations sufficiently moisten the vortex of CNTL to intensify approximately at the observed RI rate of Soudelor. These results further indicate that assimilation of all-sky satellite BTs is shown to be able to constrain the inner-core moisture and related convection.

Despite using the same initial TC vortex, there is a large difference in TC intensity forecasts between experiments such as CNTL, VentlQqonly, VentlQqoff, and VcntlQqoff + cntl_awn0. These differences, as large as $30 \mathrm{hPa}$ or $15 \mathrm{~m} \mathrm{~s}^{-1}$, are due mostly to whether or not water species in the state variables are updated in the EnKF assimilation of all-sky radiances. This result, in turn, further demonstrates the importance of moisture initialization as a significant source of forecast uncertainty, which is consistent with recent findings of Emanuel and Zhang (2017). Moreover, VqoffQcntl and VqonlyQcntl, which have the same initial moisture content but with quite different initial vortex structure and intensity, also undergo quite different intensity evolutions. Better initialization of both atmospheric moisture and vortex initial conditions is necessary to simulate the inner-core development with enhanced convective activity, and thus the RI onset of Soudelor. Although moist convection is chaotic in nature with limited predictability, improved representation of innercore vortex, water vapor, and hydrometeors is indicated 
(a) Vorticity

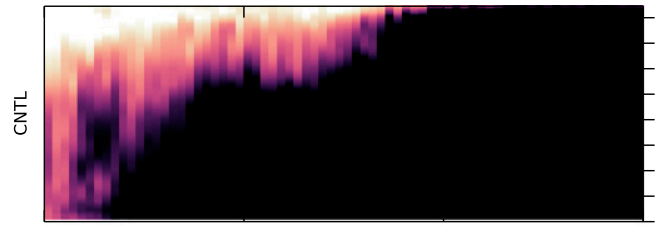

(c)

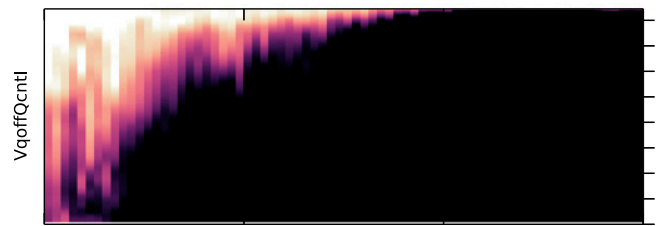

(e)

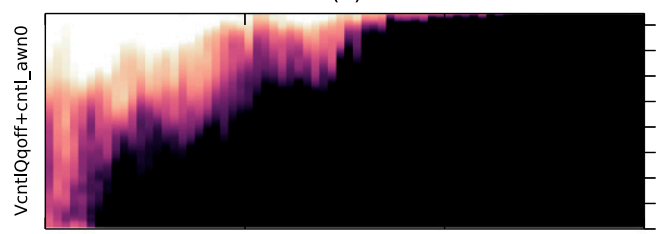

(g)

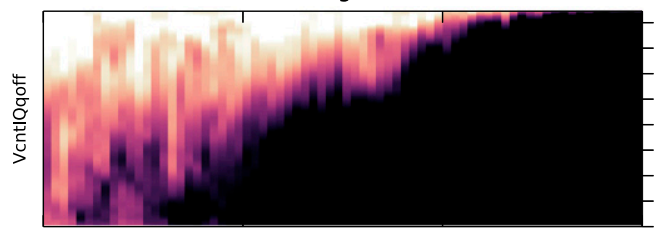

(i)
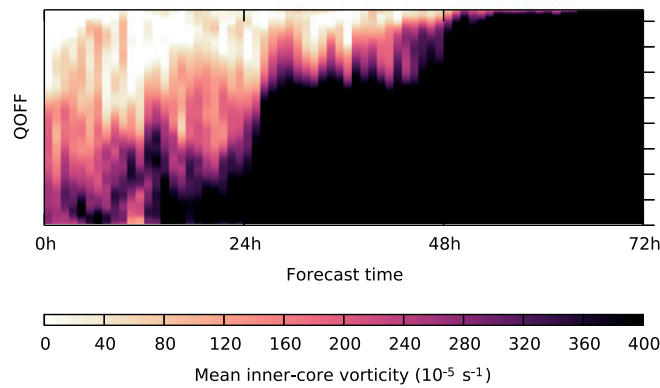

(b) Perturbed potential temperature

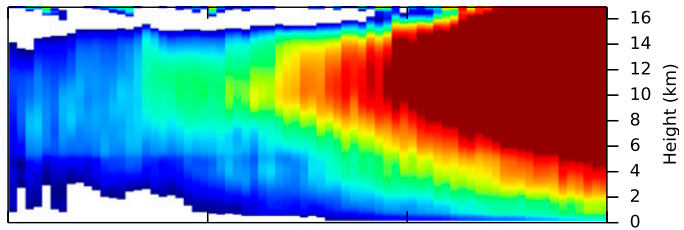

(d)

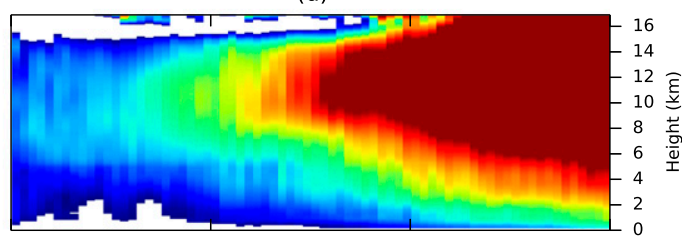

(f)

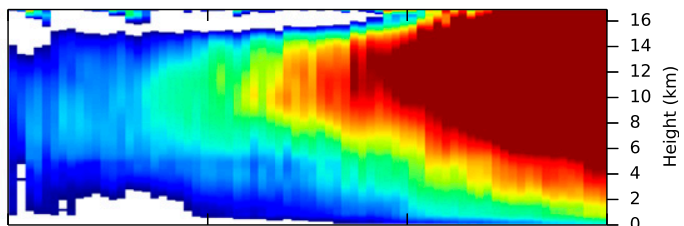

(h)

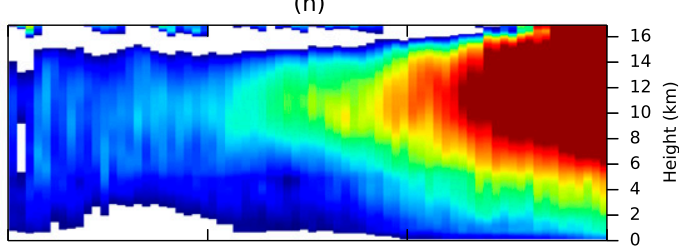

(j)
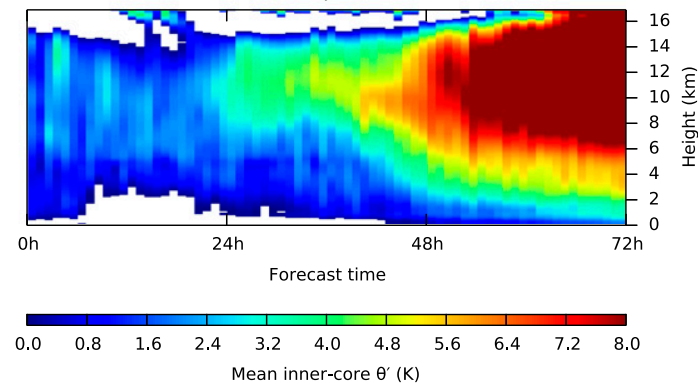

FIG. 13. Temporal evolution of inner-core (left) vorticity and (right) perturbation potential temperature from (a),(b) CNTL; (c),(d) VqoffQcntl; (e),(f) VcntlQqoff+cntl_awn0; (g),(h) VcntlQqoff; and (i),(j) QOFF. Mean inner core means the $50-\mathrm{km}$ averaged around the maximum low-pass filtered $(>200 \mathrm{~km})$ potential vorticity point at $850 \mathrm{hPa}$.

to facilitate the TC vortex development and subsequent rapid intensification.

\section{Concluding remarks}

The potential impacts of assimilating all-sky satellite brightness temperatures on tropical cyclone forecasts have been investigated through a series of convectionpermitting EnKF analysis and forecast sensitivity experiments for Supertyphoon Soudelor (2015). This study applies a set of assimilation strategies proposed for allsky satellite BTs to the real observations from the newgeneration geostationary satellite Himawari-8. The assimilation of water vapor channel $8(6.19 \mu \mathrm{m})$ from
Himawari-8 demonstrates promising impacts on the analysis and forecast of tropical cyclones. The assimilation of BTs using the EnKF leads to improved analysis of the distribution of convection embedded in the tropical depression. The deterministic forecasts based on this analysis are able to capture Soudelor's rapid intensification. The sensitivity experiments in which waterand non-water-related variables are not updated during the assimilation of BTs reveal the importance of better representing both the vortex and moisture through satellite BT assimilation. Assimilation of BTs helps to constrain convective activity, as well as to develop a more resilient TC vortex. The timing of rapid intensification (RI) onset and matured intensity is found 
to be sensitive to moisture initializations comparably with vortex initializations. Although the predictability of convection is relatively limited compared to vortex-scale flow, BT assimilation enables the occurrence of convection for the first couple of hours to be better captured. The impacts of inner-core convection are accumulated within the vortex, acting to further constrain subsequent convective activity that eventually leads to RI onset.

Numerous studies have pointed out the chaotic nature of convection as a source of forecast uncertainty for RI of TCs. While some idealized case studies have shown the strong sensitivity of RI even to almost undetectably tiny differences in moisture initializations (e.g., Emanuel and Zhang 2017; Tao and Zhang 2014; Zhang and Tao 2013), the sensitivity experiment in this study, in which the axisymmetric component of the moisture field within the TC vortex was changed, suggests the importance of the azimuthal wavenumber- 0 moisture structure in the midtroposphere and boundary layer. However, the distribution of moisture, and its potential impacts, might vary greatly from case to case. Further investigation of the processes of moist convection occurring within a vortex, with respect to the moisture initialization as well as to the vortex structure, is required. These include, but not limited to, comparing the difference with ensemble analysis and forecast uncertainties that will allow us to evaluate the significance of the impacts of moisture initializations. More systematic analysis with a large number of cases will be conducted to reveal the necessary phenomena to accurately simulate the RI onset, and to make more advances in TC forecasting with the assimilation of all-sky infrared BT.

Acknowledgments. This research is partially supported by NSF Grant 1305798, ONR Grant N000140910526, and NASA Grants NNX16AD84G and NNX15AQ51G. MM was also supported by Japan's Funai Overseas Scholarship of the Funai Foundation for Information Technology. We benefited from discussions with Eugene Clothiaux, Yonghui Weng, Michael Ying, Scott Sieron, and many others. Insightful comments from three anonymous reviewers and thorough proofreading by Robert Nystrom were greatly beneficial. Computing was provided by the Texas Advanced Computing Center (TACC). All data presented are stored and can be accessed through the TACC data archive.

\section{REFERENCES}

Aberson, S. D., A. Aksoy, K. J. Sellwood, T. Vukicevic, and X. Zhang, 2015: Assimilation of high-resolution tropical cyclone observations with an ensemble Kalman filter using HEDAS: Evaluation of 2008-11 HWRF forecasts. Mon. Wea. Rev., 143, 511-523, https://doi.org/10.1175/MWR-D-14-00138.1.

Barker, D. M., W. Huang, Y.-R. Guo, A. J. Bourgeois, and Q. N. Xiao, 2004: A three-dimensional variational data assimilation system for MM5: Implementation and initial results. Mon. Wea. Rev., 132, 897-914, https://doi.org/10.1175/1520-0493(2004) 132<0897:ATVDAS>2.0.CO;2.

Emanuel, K., 1995: Sensitivity of tropical cyclones to surface exchange coefficients and a revised steady-state model incorporating eye dynamics. J. Atmos. Sci., 52, 3969-3976, https:// doi.org/10.1175/1520-0469(1995)052<3969:SOTCTS >2.0.CO;2. , and F. Zhang, 2016: On the predictability and error sources of tropical cyclone intensity forecasts. J. Atmos. Sci., 73, 37393747, https://doi.org/10.1175/JAS-D-16-0100.1.

, and - 2017: The role of inner-core moisture in tropical cyclone predictability and practical forecast skill. J. Atmos. Sci., 74, 2315-2324, https://doi.org/10.1175/JAS-D-17-0008.1.

Han, Y., P. van Delst, Q. Liu, F. Weng, B. Yan, R. Treadon, and J. Derber, 2006: JCSDA Community Radiative Transfer Model (CRTM), version 1. NOAA Tech. Rep. NESDIS 122, 31 pp., http://docs.lib.noaa.gov/noaa_documents/NESDIS/ TR_NESDIS/TR_NESDIS_122.pdf.

— , F. Weng, Q. Liu, and P. van Delst, 2007: A fast radiative transfer model for SSMIS upper atmosphere sounding channels. J. Geophys. Res., 112, D11121, https://doi.org/10.1029/ 2006JD008208.

Harnisch, F., M. Weissmann, and Á. Periáñez, 2016: Error model for the assimilation of cloud-affected infrared satellite observations in an ensemble data assimilation system. Quart. J. Roy. Meteor. Soc., 142, 1797-1808, https://doi.org/10.1002/qj.2776.

Heidinger, A., C. O'Dell, R. Bennartz, and T. Greenwald, 2006: The successive-order-of-interaction radiative transfer model. Part I: Model development. J. Appl. Meteor. Climatol., 45, 1388-1403, https://doi.org/10.1175/JAM2387.1.

Honda, T., and Coauthors, 2018: Assimilating all-sky Himawari-8 satellite infrared radiances: A case of Typhoon Soudelor (2015). Mon. Wea. Rev., 146, 213-229, https://doi.org/10.1175/ MWR-D-16-0357.1.

Hong, S.-Y., and J. Lim, 2006: The WRF single-moment 6-class microphysics scheme (WSM6). J. Korean Meteor. Soc., 42, 129-151.

—, Y. Noh, and J. Dudhia, 2006: A new vertical diffusion package with an explicit treatment of entrainment processes. Mon. Wea. Rev., 134, 2318-2341, https://doi.org/10.1175/ MWR3199.1.

Iacono, M. J., J. S. Delamere, E. J. Mlawer, M. W. Shephard, S. A. Clough, and W. D. Collins, 2008: Radiative forcing by longlived greenhouse gases: Calculations with the AER radiative transfer models. J. Geophys. Res., 113, D13103, https://doi.org/ 10.1029/2008JD009944.

Jones, T. A., J. A. Otkin, D. J. Stensrud, and K. Knopfmeier, 2013: Assimilation of satellite infrared radiances and Doppler radar observations during a cool season observing system simulation experiment. Mon. Wea. Rev., 141, 3273-3299, https://doi.org/ 10.1175/MWR-D-12-00267.1.

,,--- , and,- 2014 : Forecast evaluation of an observing system simulation experiment assimilating both radar and satellite data. Mon. Wea. Rev., 142, 107-124, https:// doi.org/10.1175/MWR-D-13-00151.1.

Minamide, M., and F. Zhang, 2017: Adaptive observation error inflation for assimilating all-sky satellite radiance. Mon. Wea. Rev., 145, 1063-1081, https://doi.org/10.1175/ MWR-D-16-0257.1.

Munsell, E. B., F. Zhang, F. Sippel, S. A. Braun, and Y. Weng, 2017: Dynamics and predictability of the intensification of Hurricane Edouard (2014). J. Atmos. Sci., 74, 573-595, https:// doi.org/10.1175/JAS-D-16-0018.1. 
Olander, T. L., and C. S. Velden, 2007: The advanced Dvorak technique: Continued development of an objective scheme to estimate tropical cyclone intensity using geostationary infrared satellite imagery. Wea. Forecasting, 22, 287-298, https:// doi.org/10.1175/WAF975.1.

Otkin, J., 2010: Clear and cloudy sky infrared brightness temperature assimilation using an ensemble Kalman filter. J. Geophys. Res., 115, D19207, https://doi.org/10.1029/ 2009JD013759.

_ 2012: Assimilation of water vapor sensitive infrared brightness temperature observations during a high impact weather event. J. Geophys. Res., 117, D19203, https://doi.org/10.1029/ 2012JD017568.

Rappin, E. D., D. S. Nolan, and S. J. Majumdar, 2013: A highly configurable vortex initialization method for tropical cyclones. Mon. Wea. Rev., 141, 3556-3575, https://doi.org/10.1175/ MWR-D-12-00266.1.

Skamarock, W. C., J. B. Klemp, J. Dudhia, D. O. Gill, D. M. Barker, W. Wang, and J. G. Powers, 2008: A description of the Advanced Research WRF version 2. NCAR Tech. Note NCAR/TN-468+STR, 88 pp., https://doi.org/10.5065/ D6DZ069T

Tao, D., and F. Zhang, 2014: Effect of environmental shear, seasurface temperature and ambient moisture on the formation and predictability of tropical cyclones: An ensemble-mean perspective. J. Adv. Model. Earth Syst., 6, 384-404, https:// doi.org/10.1002/2014MS000314.

, and - 2015: Effects of vertical wind shear on the predictability of tropical cyclones: Practical versus intrinsic limit. J. Adv. Model. Earth Syst., 7, 1534-1553, https://doi.org/ 10.1002/2015MS000474.

Tiedtke, M., 1989: A comprehensive mass flux scheme for cumulus parameterization in large-scale models. Mon. Wea. Rev., 117, 1779-1800, https://doi.org/10.1175/1520-0493(1989)117<1779: ACMFSF $>2.0 . \mathrm{CO} ; 2$.

Vukicevic, T., and M. Sengupta, 2006: Cloud-resolving satellite data assimilation: Information content of IR window observations and uncertainties in estimation. J. Atmos. Sci., 63, 901-919, https://doi.org/10.1175/JAS3639.1.

, T. Greenwald, M. Zupanski, D. Zupanski, T. Vonder Haar, and A. S. Jones, 2004: Mesoscale cloud state estimation from visible and infrared satellite radiances. Mon. Wea. Rev., 132, 3066-3077, https://doi.org/10.1175/MWR2837.1.

Wang, P., and Coauthors, 2015: Assimilation of thermodynamic information from advanced infrared sounders under partially cloudy skies for regional NWP. J. Geophys. Res. Atmos., 120 , 5469-5484, https://doi.org/10.1002/2014JD022976.

Weng, F., 2007: Advances in radiative transfer modeling in support of satellite data assimilation. J. Atmos. Sci., 64, 3799-3807, https://doi.org/10.1175/2007JAS2112.1.

Weng, Y., and F. Zhang, 2012: Assimilating airborne Doppler radar observations with an ensemble Kalman filter for convection-permitting hurricane initialization and prediction: Katrina (2005). Mon. Wea. Rev., 140, 841-859, https://doi.org/ 10.1175/2011MWR3602.1. $\longrightarrow$, and — 2016: Advances in convection-permitting tropical cyclone analysis and prediction through EnKF assimilation of reconnaissance aircraft observations. J. Meteor. Soc. Japan, 94, 345-358, https://doi.org/10.2151/jmsj.2016-018.

Wu, C.-C., and Coauthors, 2005: Dropwindsonde Observations for Typhoon Surveillance near the Taiwan Region (DOTSTAR): An overview. Bull. Amer. Meteor. Soc., 86, 787-790, https:// doi.org/10.1175/BAMS-86-6-787.

—, Y.-H. Huang, and G.-Y. Lien, 2012: Concentric eyewall formation in Typhoon Sinlaku (2008). Part I: Assimilation of T-PARC data based on the ensemble Kalman filter (EnKF). Mon. Wea. Rev., 140, 506-527, https://doi.org/10.1175/ MWR-D-11-00057.1.

Zhang, F., and J. A. Sippel, 2009: Effects of moist convection on hurricane predictability. J. Atmos. Sci., 66, 1944-1961, https:// doi.org/10.1175/2009JAS2824.1.

- and D. Tao, 2013: Effects of vertical wind shear on the predictability of tropical cyclones. J. Atmos. Sci., 70, 975-983, https://doi.org/10.1175/JAS-D-12-0133.1.

—_, and Y. Weng, 2015: Predicting hurricane intensity and associated hazards: A five-year real-time forecast experiment with assimilation of airborne Doppler radar observations. Bull. Amer. Meteor. Soc., 96, 25-33, https://doi.org/ 10.1175/BAMS-D-13-00231.1.

— , and K. Emanuel, 2016: On the role of surface fluxes and WISHE in tropical cyclone intensification. J. Atmos. Sci., 73, 2011-2019, https://doi.org/10.1175/JAS-D-16-0011.1.

- C. Snyder, and J. Sun, 2004: Impacts of initial estimate and observation availability on convective-scale data assimilation with an ensemble Kalman filter. Mon. Wea. Rev., 132, 12381253, https://doi.org/10.1175/1520-0493(2004)132<1238: IOIEAO $>2.0 . \mathrm{CO} ; 2$.

—, Y. Weng, J. A. Sippel, Z. Meng, and C. H. Bishop, 2009: Cloud-resolving hurricane initialization and prediction through assimilation of Doppler radar observations with an ensemble Kalman filter. Mon. Wea. Rev., 137, 2105-2125, https://doi.org/10.1175/2009MWR2645.1.

,-- J. F. Gamache, and F. D. Marks, 2011: Performance of convection-permitting hurricane initialization and prediction during 2008-2010 with ensemble data assimilation of innercore airborne Doppler radar observations. Geophys. Res. Lett., 38, L15810, https://doi.org/10.1029/2011GL048469.

_, M. Minamide, and E. E. Clothiaux, 2016: Potential impacts of assimilating all-sky infrared satellite radiances from GOES-R on convection-permitting analysis and prediction of tropical cyclones. Geophys. Res. Lett., 43, 2954-2963, https://doi.org/ 10.1002/2016GL068468.

Zou, X., F. Weng, B. Zhang, L. Lin, Z. Qin, and V. Tallapragada, 2013: Impacts of assimilation of ATMS data in HWRF on track and intensity forecasts of 2012 four landfall hurricanes. J. Geophys. Res. Atmos., 118, $11558-11576$, https://doi.org/ 10.1002/2013JD020405.

, Z. Qin, and Y. Zheng, 2015: Improved tropical storm forecasts with GOES-13/15 imager radiance assimilation and asymmetric vortex initialization in HWRF. Mon. Wea. Rev., 143, 2485-2505, https://doi.org/10.1175/MWR-D-14-00223.1. 